\title{
Magnetism Science with the Square Kilometre Array
}

\author{
George Heald ${ }^{1, *}\left(\mathbb{C}\right.$, Sui Ann Mao ${ }^{2}$, Valentina Vacca ${ }^{3}{ }^{\oplus}$, Takuya Akahori ${ }^{4}(\mathbb{D}$, \\ Ancor Damas-Segovia ${ }^{5}{ }^{(0}$, B. M. Gaensler ${ }^{6}{ }^{(}$, Matthias Hoeft ${ }^{7}$, Ivan Agudo ${ }^{5}{ }^{(0}$, Aritra Basu ${ }^{8}{ }^{\circ}$, \\ Rainer Beck ${ }^{2}$, Mark Birkinshaw ${ }^{9}$, Annalisa Bonafede ${ }^{10,11}$, Tyler L. Bourke ${ }^{12}{ }^{1}$,

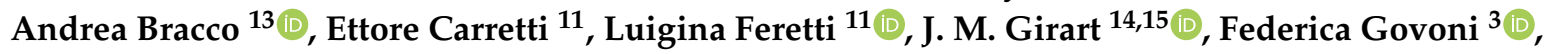 \\ James A. Green ${ }^{1}{ }^{\circ}$, Jinlin Han ${ }^{16,17,18} \oplus$, Marijke Haverkorn ${ }^{19} \oplus$, Cathy Horellou ${ }^{20} \oplus$, \\ Melanie Johnston-Hollitt ${ }^{21}{ }^{10}$, Roland Kothes ${ }^{22}$, Tom Landecker ${ }^{22}$, \\ Błażej Nikiel-Wroczyński ${ }^{23}{ }^{-}$, Shane P. O'Sullivan ${ }^{24}{ }^{(0}$, Marco Padovani ${ }^{25}{ }^{(1)}$, \\ Frédérick Poidevin ${ }^{26,27}\left(\mathbb{C}\right.$, Luke Pratley ${ }^{6}$, Marco Regis ${ }^{28,29}{ }^{\circledR}$, Christopher John Riseley ${ }^{10,11}{ }^{(0)}$, \\ Tim Robishaw ${ }^{22}{ }^{(\mathbb{D}}$, Lawrence Rudnick $\left.{ }^{30}{ }^{(}\right)$, Charlotte Sobey $\left.{ }^{1}{ }^{(}\right)$, Jeroen M. Stil ${ }^{31}{ }^{\circledR}$, \\ Xiaohui Sun ${ }^{32}{ }^{\circledR}$, Sharanya Sur ${ }^{33}$, A. Russ Taylor ${ }^{34,35,36}$, Alec Thomson ${ }^{1}{ }^{1}$, \\ Cameron L. Van Eck ${ }^{6}\left(\mathbb{D}\right.$, Franco Vazza ${ }^{10,11}$, Jennifer L. West ${ }^{6}(\mathbb{D}$ \\ and the SKA Magnetism Science Working Group ${ }^{\dagger}$
}

1 CSIRO Astronomy and Space Science, P.O. Box 1130, Bentley, WA 6102, Australia; james.green@csiro.au (J.A.G.); charlotte.sobey@csiro.au (C.S.); alec.thomson@csiro.au (A.T.)

2 Max Planck Institute for Radio Astronomy, Auf dem Hügel 69, 53121 Bonn, Germany; mao@mpifr-bonn.mpg.de (S.A.M.); rbeck@mpifr-bonn.mpg.de (R.B.)

3 INAF-Osservatorio Astronomico di Cagliari, Via della Scienza 5, 09047 Selargius, Italy; valentina.vacca@inaf.it (V.V.); federica.govoni@inaf.it (F.G.)

4 Mizusawa VLBI Observatory, National Astronomical Observatory of Japan (NAOJ), 2-21-1 Osawa, Mitaka, Tokyo 181-8588, Japan; takuya.akahori@nao.ac.jp

5 Instituto de Astrofísica de Andalucía (CSIC), Glorieta de la Astronomía, 18008 Granada, Spain; adamas@iaa.es (A.D.-S.); iagudo@iaa.es (I.A.)

6 Dunlap Institute for Astronomy and Astrophysics, University of Toronto, 50 St George Street, Toronto, ON M5S 3H4, Canada; bgaensler@dunlap.utoronto.ca (B.M.G.); luke.pratley@dunlap.utoronto.ca (L.P.); cameron.van.eck@dunlap.utoronto.ca (C.L.V.E.); jennifer.west@dunlap.utoronto.ca (J.L.W.)

7 Thüringer Landessternwarte, Sternwarte 5, 07778 Tautenburg, Germany; hoeft@tls-tautenburg.de

8 Fakultät für Physik, Universität Bielefeld, Postfach 100131, 33501 Bielefeld, Germany; aritra@physik.uni-bielefeld.de

9 HH Wills Physics Laboratory, University of Bristol, Tyndall Avenue, Bristol BS8 1TL, UK; mark.birkinshaw@bristol.ac.uk

10 Dipartimento di Fisica e Astronomia, Università degli Studi di Bologna, via P. Gobetti 93/2, 40129 Bologna, Italy; annalisa.bonafede@unibo.it (A.B.); christopher.riseley@unibo.it (C.J.R.); franco.vazza2@unibo.it (F.V.)

11 INAF-Istituto di Radioastronomia, Via Gobetti 101, 40129 Bologna, Italy; ettore.carretti@inaf.it (E.C.); lferetti@ira.inaf.it (L.F.)

12 SKA Organization, Jodrell Bank, Lower Withington, Macclesfield SK11 9FT, UK; t.bourke@skatelescope.org Rudjer Bošković Institute, Bijenička cesta 54, 10000 Zagreb, Croatia; abracco@irb.hr

4 Institut de Ciencies de l'Espai (CSIC), 08193 Cerdanyola del Vallès, Catalonia, Spain; girart.astro@gmail.com 5 Institut d'Estudis Espacials de Catalunya (IEEC), 08034 Barcelona, Catalonia, Spain

16 National Astronomical Observatories, Chinese Academy of Sciences, Jia-20 DaTun Road, ChaoYang District, Beijing 100101, China; hjl@nao.cas.cn

17 School of Astronomy, University of Chinese Academy of Sciences, Beijing 100049, China

18 CAS Key Laboratory of FAST, NAOC, Chinese Academy of Sciences, Beijing 100101, China

19 Department of Astrophysics/IMAPP, Radboud University Nijmegen, P.O. Box 9010, 6500 GL Nijmegen, The Netherlands; m.haverkorn@astro.ru.nl

20 Chalmers University of Technology, Dept of Space, Earth and Environment, Onsala Space Observatory, 43992 Onsala, Sweden; Cathy.Horellou@chalmers.se

21 International Centre for Radio Astronomy Research, Curtin University, Bentley, WA 6102, Australia; melanie.johnston-hollitt@curtin.edu.au 
22 National Research Council Canada, Herzberg Programs in Astronomy \& Astrophysics, Dominion Radio Astrophysical Observatory, P.O. Box 248, Penticton, BC V2A 6J9, Canada; roland.kothes@nrc-cnrc.gc.ca (R.K.); tom.landecker.drao@gmail.com (T.L.); tim.robishaw@gmail.com (T.R.) Astronomical Observatory of the Jagiellonian University, ul. Orla 171, 30-244 Kraków, Poland; blazej.nikiel_wroczynski@uj.edu.pl

24 Centre for Astrophysics and Relativity, Dublin City University, Glasnevin, Ireland; shane.osullivan@dcu.ie

25 INAF-Osservatorio Astrofisico di Arcetri, Largo E. Fermi 5, 50125 Firenze, Italy; padovani@arcetri.astro.it

26 Instituto de Astrofisíca de Canarias, 38200 La Laguna, Tenerife, Spain; fpoidevin@iac.es

27 Departamento de Astrofísica, Universidad de La Laguna (ULL), 38206 La Laguna, Tenerife, Spain

28 Dipartimento di Fisica, Università degli Studi di Torino, Via P. Giuria 1, 10125 Torino, Italy; regis.mrc@gmail.com

29 INFN-Istituto Nazionale di Fisica Nucleare, Sezione di Torino, Via P. Giuria 1, 10125 Torino, Italy

30 Minnesota Institute for Astrophysics, School of Physics and Astronomy, University of Minnesota, 116 Church Street SE, Minneapolis, MN 55455, USA; larry@umn.edu

31 Department of Physics and Astronomy, The University of Calgary, 2500 University Drive NW, Calgary, AB T2N 1N4, Canada; jstil@ucalgary.ca

32 Department of Astronomy, Yunnan University, and Key Laboratory of Astroparticle Physics of Yunnan Province, Kunming 650091, China; xiaohui.sun112@gmail.com

33 Indian Institute of Astrophysics, 2nd Block, Koramangala, Bangalore 560034, India; sharanya.sur@iiap.res.in

34 Department of Astronomy, University of Cape Town, Private Bag X3, Rondebosch 7701, South Africa; russ@idia.ac.za

35 Department of Physics and Astronomy, University of the Western Cape, Private Bag X17, Bellville 7535, South Africa

36 Inter-University Institute for Data Intensive Astronomy, Private Bag X3, Rondebosch 7701, South Africa

* Correspondence: george.heald@csiro.au; Tel.: +61-(0)8-6436-8758

+ The SKA Magnetism Science Working Group, Jodrell Bank, Lower Withington, Macclesfield, Cheshire SK11 9FT, UK.

Received: 30 April 2020; Accepted: 4 June 2020; Published: 6 July 2020

Abstract: The Square Kilometre Array (SKA) will answer fundamental questions about the origin, evolution, properties, and influence of magnetic fields throughout the Universe. Magnetic fields can illuminate and influence phenomena as diverse as star formation, galactic dynamics, fast radio bursts, active galactic nuclei, large-scale structure, and dark matter annihilation. Preparations for the SKA are swiftly continuing worldwide, and the community is making tremendous observational progress in the field of cosmic magnetism using data from a powerful international suite of SKA pathfinder and precursor telescopes. In this contribution, we revisit community plans for magnetism research using the SKA, in light of these recent rapid developments. We focus in particular on the impact that new radio telescope instrumentation is generating, thus advancing our understanding of key SKA magnetism science areas, as well as the new techniques that are required for processing and interpreting the data. We discuss these recent developments in the context of the ultimate scientific goals for the SKA era.

Keywords: magnetic fields; polarization; instrumentation: interferometers; techniques: polarimetric; telescopes

\section{Introduction}

Cosmic magnetism has traditionally been a relatively specialised field, but is increasingly recognised as a domain where new progress is crucial to gain greater understanding of broader astrophysical phenomena such as the star formation process, galaxy evolution, the physics of phenomena related to active galactic nuclei, galaxy clusters, and large-scale structure, and the evolution of the early Universe. Following steady progress over the last several decades through a diverse range 
of observational tracers and theoretical approaches, a leap forward in the radio domain is anticipated with the development of the Square Kilometre Array (SKA). The first phase of the SKA will comprise two interferometric radio telescope arrays: a low-frequency array in Western Australia (SKA1-LOW) observing from 50-350 MHz with 131,072 broadband log-periodic dipoles organised into 512 stations separated by a maximum baseline of $65 \mathrm{~km}$; and a mid-frequency array in South Africa (SKA1-MID) observing at least from 350-1760 and 4600-15,300 MHz with 197 offset-Gregorian antennas separated by a maximum baseline of $150 \mathrm{~km}$. A full description of the SKA "Design Baseline" is provided on the project website ${ }^{1}$.

The impact of the SKA on magnetism science was first broadly considered in a volume dedicated to the SKA science case about 16 years ago [1-4]. Since then, the SKA Organisation has fostered the development of this science case (and others) within the wide international community through the Cosmic Magnetism Science Working Group (SWG). This SWG was instrumental in developing an updated description of SKA science ambitions, as was comprehensively described about five years ago in the two-volume SKA Science Book ${ }^{2}$. An overview of chapters within the field of cosmic magnetism, as well as a description of the headline magnetism "Rotation Measure (RM) Grid" survey were provided [5]. The overview chapter was supplemented with additional detailed contributions in the areas of active galactic nuclei and radio galaxies [6-9], nearby galaxies [10,11], galaxy clusters [12-14], the Milky Way [15,16], the interstellar medium (ISM) and star formation [17,18], large-scale structure and the cosmic web [19,20], dark matter [21], and advanced techniques [22-24].

Since then, rapid progress in developing our understanding of cosmic magnetism and refining the questions that we will seek to answer with the SKA is already visible based on results that are now starting to flow from SKA pathfinder and precursor activities. This paper seeks to provide an updated snapshot view of the field, on the basis of presentations and discussions at the most recent SKA science meeting ${ }^{3}$, which was held in Manchester (U.K.) from 8-12 April 2019.

The radio domain ${ }^{4}$ has been very successful for many years in tracing the magnetic fields that pervade the Universe on all physical scales $[25,26]$. Three primary techniques are employed: (i) synchrotron radiation, including its degree of linear and circular polarization; (ii) the Faraday rotation effect induced by intervening magneto-ionic media; and (iii) Zeeman splitting of energy levels in atoms and molecules. A full review of these mechanisms is beyond the scope of this paper; we refer the reader to [27] for a detailed description of the insights that can be gained from the study of synchrotron emission and Faraday rotation and [28,29] for reviews of the power of Zeeman splitting.

This paper is organised as follows. We begin by revisiting the key science areas in the field of cosmic magnetism that we intend to probe with the SKA (Section 2), highlighting advances that have occurred since the publication of the 2015 SKA science book. This progress is now rapidly taking place with an impressive international suite of SKA precursor and pathfinder telescopes (Section 3), while simultaneously clarifying the capabilities and potential of those instruments. These ongoing developments are not only answering existing scientific questions and prompting new ones, but also serving to clarify the technical considerations that are required to maximise the science achievable using SKA observations (Section 4) and providing a fresh context for the design of the key surveys anticipated to be delivered by the SKA (Section 5). We conclude the paper with some thoughts about the steps that will be required in the coming years before the SKA enters its operational phase (Section 6).

\section{SKA Magnetism Science Cases}

Cosmic magnetism has been a key SKA science driver since the early planning phases of the project [30], including an early "RM Grid" concept (e.g., [1]). The SKA holds great promise because

\footnotetext{
https:/ / astronomers.skatelescope.org/documents /

https://pos.sissa.it/cgi-bin/reader/conf.cgi?confid=215

https: / /indico.skatelescope.org/event/467/

In this paper, we focus primarily on the frequency range intended to be covered by the SKA: $50 \mathrm{MHz} \leq v \leq 24 \mathrm{GHz}$.
} 
of the central importance of meter- and centimetre-wavelength radio observations for the study of cosmic magnetism. Synchrotron radiation, which traces magnetic fields perpendicular to the line of sight, is typically dominant at these wavelengths. Synchrotron radiation is intrinsically linearly and circularly polarized at a fractional amount that reflects the degree of order of the magnetic field. Faraday rotation and depolarization effects, as well as the conversion of linear into circular polarization (e.g., [31,32]), which depend on the magnetic field strength along the line of sight, are observable effects that typically carry detailed behaviour across broad frequency ranges, reflecting the structure of the magneto-ionic medium. Furthermore, the Zeeman effect that is observed in molecular lines such as $\mathrm{OH}, \mathrm{H}_{2} \mathrm{O}$, and to a lesser extent, $\mathrm{CH}_{3} \mathrm{OH}$ (as a non-paramagnetic molecule), provides us with an independent measurement of in situ magnetic fields threading molecular clouds.

The magnetism-oriented chapters ${ }^{5}$ of the 2015 SKA Science Book (see [5] for an overview) describe how modern techniques will be revolutionary for developing a newly detailed observational picture of cosmic magnetism. Specifically, the community now makes use of Faraday rotation measure (RM) synthesis (originally conceived by [33], and updated in the context of modern radio telescope technology by [34]) and the complementary analysis technique "Faraday tomography", through which observed features are associated with structure along the line of sight. This approach is based on the Faraday depth [33]:

$$
\phi(r) \propto \int_{r}^{0} n_{e} \vec{B} \cdot \mathrm{d} \vec{l}
$$

where $n_{e}$ is the thermal electron density, $\vec{B}$ is the magnetic field, $\vec{l}$ is the line of sight, and the sign convention is that $\phi$ is positive for $\vec{B}$ directed toward the observer. Although Faraday depth is not equivalent to physical distance, expressing linear polarization as a function of this quantity provides the potential to distinguish multiple magneto-ionic contributions along the line of sight to and through radio sources. However, interpretation of the observed features in a three-dimensional distribution is not a trivial task and requires complementary multi-wavelength observations, simulations, and other tools for analysis and interpretation. During the last several years, the power of RM synthesis and Faraday tomography has been demonstrated in various aspects of cosmic magnetism (see for example [35]), thanks in large part to the wider wavelength coverage of modern radio telescopes. Although Faraday rotation measure and Faraday depth provide combined information about the line of sight magnetic field, thermal gas density, and distance, supplementary constraints on the thermal gas density and distance can be provided by multi-wavelength observations in the X-ray and optical bands, respectively, thus isolating and constraining the magnetic field contribution. For impulsive sources such as pulsars and fast radio bursts (FRBs), the dispersion measure (DM $\propto \int n_{e} \mathrm{~d} l$ ) also provides complementary information about the thermal electron density along the line of sight (e.g., [36]). This multi-wavelength and multi-tracer observational approach has started to take shape through the efforts of various research groups over the past few years.

In this section, we provide brief reviews of the primary magnetism science cases that have been developing, with an emphasis on recent progress that has been made in theoretical and observational studies. The study of cosmic magnetism spans diverse astrophysical domains, and magnetic fields within the corresponding observational targets range over a wide variety of scales, from Mpc down to sub-pc. Overarching these detailed studies are two common questions: (1) What is the origin and evolution of magnetic fields throughout the Universe? (2) How do magnetic fields illuminate and influence the physical processes in different objects? The SKA will probe magneto-ionic media in many different environments including the large-scale structure of the Universe and the intergalactic medium (IGM), large-scale jets and outflows from AGN, the formation and evolution of galaxies and stars, and the properties of the interstellar medium (ISM).

5 https://pos.sissa.it/cgi-bin/reader/ conf.cgi? confid=215\#session-2111 


\subsection{Large-Scale Structure and Cosmology}

\subsubsection{The Cosmic Web}

The largest structures in the Universe comprise the cosmic web, the network of filaments and sheets that connects galaxy clusters. Constraining the properties of the magnetic field in the cosmic web is at the frontier of cosmic magnetism research. It has been predicted by numerical simulations that the cosmic web is filled with baryons at temperatures ranging from $10^{5} \mathrm{~K}$ to $10^{7} \mathrm{~K}$. This warm-hot IGM, or WHIM, is the main candidate for the solution to the so-called "missing baryon problem" $[37,38]$ in the local Universe and is cospatial with the intergalactic magnetic field (IGMF). If the IGMF was formed in the early Universe (Section 2.1.2), then its presence can be best traced in voids or on the largest linear scales $(>10 \mathrm{Mpc})$ in the cosmic web. While in the case of galaxy clusters, the presence of significant dynamo amplification of primordial (or galactic) seed fields is beyond doubt, in the IGMF of filaments, the level of dynamo amplification and the memory of ancient magnetic seeding events is more debated, and ultimately related to the (unknown) level of plasma turbulence there.

The community is seeking detections of the filamentary magnetised cosmic web through two primary techniques: diffuse, low surface brightness synchrotron emission originating from within the filaments themselves and through careful analysis of RMs for background sources with lines of sight passing through the large-scale structure. Recently, a number of radio observations have attempted to constrain the IGMF strength in filaments [39-43]. RMs from extragalactic polarized sources and fast radio bursts (FRBs; Section 2.1.5) have been used to place limits on the strength of the IGMF in voids of less than $10 \mathrm{nG}$ [44-48], which already rules out some of the theoretical predictions [49-51]. In order to put stringent constraints on the IGMF on the basis of RMs for extragalactic polarized sources, a detailed knowledge of the thermal gas density distribution along the line of sight and its mixing with the magnetic field is required. Moreover, given the likely low level of RMs from the IGMF in filaments, a careful selection of background sources and excellent sensitivity will be necessary to disentangle the intrinsic contribution from background sources from that of intervening filaments (e.g., [52]), in addition to carefully separating the Galactic RM foreground. The prospect of constructing RM Grids using FRBs is of particular interest due to the simultaneous availability of rotation and dispersion measures [50,53]. The SKA will discover hundreds to thousands of localised FRBs [54] and therefore deliver RM Grids from FRBs, which have the potential to untangle the degeneracy between electron density and magnetic field.

\subsubsection{Early Universe and the Epoch of Reionization}

In the early Universe, primordial magnetogenesis scenarios may require some modifications to the standard theory of inflation and cosmology; such magnetic fields could impact the formation of the large-scale structure in the Universe [55]. The existence of a pervasive IGMF would introduce non-Gaussian anisotropies in the CMB and could affect the duration of the Epoch of Reionization (EoR) [56,57], while the EoR itself may be crucial for seeding the IGMF [58]. Therefore, observational constraints on the IGMF in the early Universe are an important component for cosmological models. The IGMF in the early Universe may be constrained by the EoR observations that will be undertaken with the SKA [59], because magnetic fields can alter the expected spatial fluctuations of density and temperature through heating mechanisms from ambipolar diffusion and decay of turbulence in the IGM [60].

\subsubsection{Galaxy Clusters}

Magnetic fields in clusters have historically been revealed through detection of diffuse synchrotron emission from the intracluster medium (ICM) and Faraday RMs of embedded and background radio sources. The number of diffuse radio sources identified in galaxy clusters has increased by a few hundred over the past few years [61], largely due to an increase in the prevalence of high-quality observations at low radio frequencies. Radio halos are found to be generally unpolarized, likely due 
to depolarization effects, limited resolution in Faraday space, and because current interferometers have limited sensitivity and angular resolution. Based on the existence of $\mu \mathrm{G}$-level magnetic fields at cluster centres, intrinsic polarization of radio halos is expected at 15-35\% levels [62]. Detection of this emission will provide information about the ICM magnetic field power spectrum.

It remains unclear how magnetic fields are distributed and amplified during cluster evolution and how cosmic rays are (re-)accelerated in this environment. These processes are tightly coupled to the physics of shock waves and magneto-hydrodynamic (MHD) turbulence in the ICM (e.g., [63,64]). During the formation of galaxy clusters, up to $10^{64}$ ergs are deposited in the ICM on large spatial scales and then on increasingly smaller scales through turbulent cascades. The strength and coherence length of cluster magnetic fields should depend on the growth stage of MHD turbulence, implying that they vary between merging clusters, regular clusters, and cool-core clusters. The mechanism of growth of intracluster magnetic fields is still debated. Initial simulations by [65] have shown that its strength saturates during cluster formation, while later work showed that dynamo action can explain the magnetic field strength presently indicated from rotation measure observations [66].

Cool-core galaxy clusters show higher gas density than merging systems. As the plasma has a very high conductivity, to first order, the magnetic field is frozen-in, and hence, its strength is expected to be higher, implying higher rotation measure values. This expectation is confirmed by radio observations that indicate magnetic fields with strengths in cluster centres of order $\mu \mathrm{G}$ and fluctuation scales up to a few hundreds of kpc in merging systems, while observations of relaxed systems indicate magnetic field strengths in cluster centres up to order $10 \mu \mathrm{G}$ and fluctuation scales of the order of tens kpc or less (see e.g., [67]). Moreover, magnetic field strength is expected to correlate with gas density and/or temperature [68].

SKA observations of galaxy clusters will be powerful for determining cluster magnetic field properties through RM synthesis if the cluster area is sufficiently sampled by background polarized sources, and especially by diffuse polarized background radio sources to resolve field structures smaller than the separation of sources in the RM Grid (e.g., [69]). The statistics of the spatial fluctuations of the larger sources can reveal the turbulent properties of the ICM.

\subsubsection{Dark Matter}

Dark matter is a fundamental ingredient of our Universe and of structure formation models, and yet, its fundamental nature is elusive to astrophysical probes. Information on the nature and physical properties of the weakly interacting massive particle (WIMP), the leading candidate for a cosmologically relevant dark matter, can be obtained by studying the astrophysical signals of their annihilation/decay. Among the various electromagnetic signals, electrons and positrons produced by WIMP annihilation generate synchrotron emission in the magnetized atmosphere of galaxy clusters and galaxies, which could be observed as a diffuse radio emission centred on the dark matter halo. A deep search for dark matter radio emission with the SKA in local dwarf galaxies, galaxy regions with low star formation, galaxy clusters (with offset dark matter-baryonic distribution, e.g., the Bullet cluster [70]), and studying angular correlations of the unresolved radio background can be effective in constraining the WIMP mass and annihilation cross-section [21]. These limits strongly depend on the magnetic field, which is typically poorly known in the quiescent regions of interest, i.e., in the regions where the astrophysical "background" is dim.

Studies with different telescopes have recently attempted the detection of a radio WIMP signal, including the Green Bank Telescope (GBT) [71], Australia Telescope Compact Array (ATCA) [72,73], LOw Frequency ARray (LOFAR) [74], and Murchison Widefield Array (MWA) [75]. They all obtained a null detection and derived upper limits on the dark matter interaction rate. The SKA will have the capability to determine simultaneously both the magnetic field strength in the dark matter-dominated structures and the dark matter particle properties. It has been shown that the search for WIMP radio signals with the SKA and its precursors (for example in the very relevant case of dwarf spheroidal galaxies [72]) will progressively close in on the full parameter space of WIMPs, which means it will 
constrain the dark matter annihilation cross-section below the so-called "thermal value". With the SKA, the uncertainties associated with the description of the ambient medium will dramatically decrease, reducing the astrophysical uncertainties and allowing a more precise determination of the dark matter bounds.

\subsubsection{FRBs}

The study of fast radio bursts (FRBs) has grown rapidly [76] since the first detection [77] just over a decade ago, with a particular rise in activity associated with localisation efforts [78,79] aimed at resolving outstanding questions about their origin and physical properties. Rotation measures from FRBs can be very useful to help constrain the environment of these enigmatic sources. As a remarkable example, observations of repeating FRB121102 indicated an extremely large $\mathrm{RM}$ of $\mathcal{O}\left(10^{5}\right) \mathrm{rad} \mathrm{m}^{-2}$ with a $10 \%$ variation over the course of seven months [80]. RM synthesis clearly indicated a single component of polarized emission behind a single extreme RM screen, implying an environment similar to a massive black hole or a supernova remnant. Meanwhile, FRBs showing small RMs of $\mathcal{O}(10) \mathrm{rad} \mathrm{m}^{-2}$ have proven powerful to constrain the IGMF to less than tens of $\mathrm{nG}[45,79]$ as described in Section 2.1.1. In the SKA era, the study of polarized emission from FRBs is expected to deliver further interesting information. For example, temporal variation of the polarization angle may provide an indication of the rotation of the repeating sources, while the degree of depolarization may constrain the proportions of regular and turbulent magnetic fields along the line of sight. The RM variations of repeating FRBs on different time scales can indicate the plasma transition near the source or in the intervening medium.

Extreme RMs of $\mathcal{O}\left(10^{5} \mathrm{rad} \mathrm{m}^{-2}\right)$ are likely to be found through comprehensive polarization surveys, including in the environments of high-power radio sources. More generally, the detection of sources with extreme RM values can be an exciting pathway to the discovery of exotic sources and should be taken into account when planning to optimise the SKA's capability for exploring the unknown Universe (e.g., [81]).

\subsection{Individual Galaxies}

\subsubsection{Normal and Star-Forming Galaxies}

Magnetic fields in the ISM of galaxies have an important moderating influence on the star formation process [82], and their properties and evolution are therefore integral to the formation and evolution of galaxies. A fundamental question is the origin of the large-scale coherent magnetic fields that are commonly observed in galaxies in the local Universe [25,83]. These magnetic fields are understood to have been amplified over poorly-constrained time scales from weak seed fields present at an early phase of galaxy formation [84-87]. To probe this amplification history observationally, efforts focus on studying the magnetic fields in high-redshift galaxies [88-91] and testing the galactic dynamo theory in the local Universe [92]. In the local galaxy population, it is crucial to observe objects with a wide range of properties, including differentially and solid-body rotating disks. The redshift evolution of the strength and order of galactic magnetic fields is a critical aspect to better understand the physics, as is the dependence on key properties such as the star formation rate, galactic rotation, and environment.

Broadband radio observations have proven to be very effective for probing the structure of magnetic fields in galaxies in the local Universe (e.g., [93,94]). Detailed studies of individual galaxies across a broad range of radio frequencies are crucial to probe the interaction between star formation and magnetic field properties (e.g., [95-98]). When applied to large and well-defined samples, these observations are now probing the typical magnetic structure in galaxy halos, far from the regions of active star formation [99], and thereby constraining the processes such as cosmic ray propagation that support and drive the structures at the outskirts of galaxies [100]. Current dynamo theory cannot explain certain aspects of galactic magnetic fields, for example in spiral arms and halos [92]. 
A comprehensive understanding of the structure and evolution of galactic magnetic fields can improve models of starburst-driven outflows, IGM feedback [101,102], and cosmological structure formation simulations $[103,104]$. Magnetic fields in the outermost parts of galaxies may be understood through a dense RM Grid (e.g., $[105,106])$ as will be delivered by the SKA.

Groups and pairs of galaxies are crucial for understanding the unique influence of environments that are denser than for galaxies in the field, but sparser than in clusters. For example, LOFAR's survey capability is facilitating studies of the typical properties in this regime (e.g., [107]). Intergalactic magnetic fields have been recently found in nearby galaxy pairs [108,109]. Using the SKA, we will be able to probe the magnetic fields in similar systems to uncover the influence of magnetism in small galaxy groups.

\subsubsection{Active Galactic Nuclei}

The scientific investigation of active galactic nuclei (AGN) requires an understanding of the physics of accretion onto the supermassive black hole (SMBH) and, in some cases, the launching of powerful relativistic jets that can extend beyond the host galaxy environment and deposit energy and magnetic field into the surrounding intergalactic medium (IGM). It is expected that mass accretion onto the SMBH makes the AGN a time-variable source [110], while the magnetic field in the disk enables the accretion of material through the magneto-rotational instability (MRI) [111]. Therefore, while it is difficult to place observational constraints on the magnetic field in the accretion disk, the time variability of AGN gives a clue to its properties.

Recently, the Event Horizon Telescope (EHT) has confirmed the general understanding of the standard theory of gravity and the mechanism of radiation transfer in the presence of an SMBH $[112,113]$. The next step is to detect the emission from the jet base, where the magnetic field is thought to play a key role in the formation of the relativistic jet. In this regard, polarization and RM observations at relatively high frequencies (allowing detection of the high expected RMs) are important to constrain the magnetic field geometry at the jet launching site. On much larger scales, polarization and RM observations have provided support for the theoretically-expected helical magnetic field geometry [114].

However, further downstream, there may be a transition to a less ordered field geometry, which radio observations can uniquely probe, before the jet terminates (or is disrupted) to generate the classical double-lobed radio galaxy structure. The radio lobe morphology can be classified into roughly two types, Fanaroff-Riley (FR) I and FR II. It is unknown to what extent the magnetic field of jets or of the surrounding medium affects the morphology of radio lobes. Investigating links between the lobe morphology and the state of the accretion disk can provide new discovery potential. Finally, AGN jets supply a significant amount of energy and magnetic field to the surrounding IGM [115-117] and are thus crucial for a complete understanding of the formation and evolution of the cosmic structure on large scales.

\subsection{Galactic and Sub-Galactic Scale Fields}

\subsubsection{The Milky Way}

The Milky Way is a unique object in which to understand magnetic field structures in spiral arms [36], the Galactic Center [118], and the Galactic halo [119,120] in exquisite detail. Magnetic fields affect Galactic large-scale (kpc) structures through the Parker instability [121] and the MRI [122] and alter Galactic small-scale (pc) structures through MHD turbulence. The interplay of these large-scale and small-scale magnetic field components provides unique insights into the dynamo mechanism that enhances and maintains magnetism in galaxies [123]. Furthermore, measurements of magnetic helicity can help to constrain dynamo theory [124]. Although the turbulent magnetic field component is expected to be ubiquitous in galaxies [83], it is only directly measurable in detail in the Milky Way (see, e.g., [125]), possibly also through interpretation of RM spectra (but see [126]). Understanding turbulent 
interstellar magnetic fields will allow us to gauge their influence on star formation, gas heating and dynamics, cosmic ray propagation, and other physical processes in the interstellar medium.

Interstellar objects such as supernova remnants, planetary nebulae, H IIregions, globular clusters, and gigantic radio loops are all shaped by the influence of the Galactic magnetic field (e.g., [127-130]); see also Section 2.3.2. The magnetic content of high velocity clouds (HVCs) such as the Smith Cloud [131] is important for their longevity. Characterizing magnetic fields in all of these objects yields insight into their evolution and impact on their Galactic environment. For the largest of these Galactic structures, single-dish data will be required to supplement the SKA. New all-sky surveys of the diffuse polarized emission from the Milky Way with single dish telescopes have been conducted recently (e.g., $[132,133])$. These surveys provide polarization observations at multiple frequency channels over a broad bandwidth, which allow us to utilize RM synthesis to study the properties of the Galactic magneto-ionic medium [134,135].

The Galactic magnetic field deflects ultra-high energy cosmic rays, thus concealing their origins $[136,137]$, and the polarization signatures that it causes provide significant foregrounds for extragalactic studies such as CMB polarization (e.g., [138-141]) and the Epoch of Reionization. Therefore, detailed knowledge of the magnetic field strength and structure of the Milky Way is essential for a number of extragalactic science drivers. An SKA1-MID RM Grid will provide a detailed view of the Galactic magnetic field structure (e.g., [142]), including field reversals, the halo field (e.g., $[119,120])$, and other constraints on Galactic magnetic field models, as well as magnetic fields associated with individual structures like bubbles and nebulae such as SNRs, pulsar wind nebulae, $\mathrm{H}$ II regions, and planetary nebulae. SKA1-LOW will complement SKA1-MID through facilitating broad-band polarimetry and high-precision RM measurements towards diffuse emission and objects in the Milky Way and beyond. The combination of an RM Grid and polarized diffuse emission has been demonstrated to be powerful in understanding extended structures [143] and will in the future be able to reveal small-scale properties of MHD turbulence, as well as a tomographic three-dimensional view of the magnetized ISM, with an even denser RM Grid using the SKA.

\subsubsection{The Interstellar Medium and Star Formation}

Elucidating the role of magnetic fields in the formation and evolution of molecular clouds, and in particular in regulating star formation within those clouds, is a key science goal of astronomy. In the last few decades, it has been recognized that magnetic fields play a crucial role in the mechanism of star formation through various astrophysical processes (e.g., [82,144-151]). For example, magnetic fields are linked to the energy dissipation of the ISM allowing molecular clouds to form and determining the physical condition of MHD turbulence [152-156]. A full understanding of MHD turbulence is required in order to explain the formation of dense molecular clouds. The SKA will be able to probe magnetic fields in molecular clouds and starless cores [157]. Magnetic fields provide an additional force to the ISM as a tension and an additional heating term though ambipolar diffusion. These are key factors required to understand the collapse of a dense molecular cloud to form a protostar.

The evolution of molecular clouds is partially controlled by magnetic fields, including in dense filaments, which are the main sites of star formation [158]. The role of magnetic fields in forming and shaping these is not yet clear [151], but can be resolved by determining the strength and degree of order of magnetic fields and comparing with simulations. Within molecular clouds, field strength measurements are via the Zeeman effect [28], readily observable in maser emission, but requiring very deep, pointed observations with high spectral resolution at radio frequencies for thermal emission (e.g., $\mathrm{OH}$ at 1665 and $1667 \mathrm{MHz}$ ). The Zeeman effect can be prominent in paramagnetic molecules, particularly $\mathrm{OH}$, but the nature of the emission can make measurements difficult, particularly thermal emission, for which it is small relative to the linewidth, and thus difficult to measure. Magnetic field structure within molecular clouds is thus typically measured via thermal dust emission and has been mainly an area for sub-millimetre and far-infrared observations (see, e.g., [159]), but maser emission 
studies can be productive (e.g., [160,161]). RM Grids can also be used to determine the magnetic field in molecular cloud regions, if they are sampled by a sufficient density of sources (e.g., [162,163]).

Magnetic fields collimate the molecular jets of young stellar objects (YSOs) through magnetic tension $[164,165]$. Magnetic fields are also believed to play a significant role in the formation and evolution of planet-forming disks [166,167]. The SKA will test this through sensitivity to polarized emission from large grains aligned with the magnetic field, which will also probe grain growth in these disks $[168,169]$. Magnetic fields in the ISM surrounding star-forming regions both influence the evolution of and may also be impacted by $\mathrm{H}$ II regions $[129,170]$. The SKA will be able to probe the detailed physical conditions in and around H II regions [171,172]. Supernova shock waves appear to amplify the magnetic fields of the upstream ISM significantly. The shapes, polarization, and Faraday depth characteristics of SNR can reveal the magnetic fields in the remnant and in the surrounding ISM [173,174]. Understanding the magnetic field amplification mechanism is necessary to examine the role of supernova shock waves in the origin of Galactic cosmic-rays.

Additionally, the near-Earth environment can be traced through Faraday rotation probes of coronal mass ejections (CMEs; e.g., $[175,176])$ and studies of the ionosphere through the resulting distortions in source positions (e.g., [177]) and eventually RMs (e.g., [178,179]).

\section{Pathfinder Progress}

SKA pathfinders and precursors feature new technical capabilities, as well as design enhancements in comparison with traditional radio telescopes. Many of these facilities are planning substantial new magnetism surveys, which provides us with a twofold opportunity: first, to test many of the analysis techniques that are planned for SKA data products, but on a somewhat smaller scale; and secondly, to further develop the science questions that will be addressed in the SKA era. In this section, we aim to highlight some SKA precursor and pathfinder telescopes that are driving substantial progress in the field and to capture recent results that they have produced.

\subsection{Science Capability from New Technology}

Substantial developments with three classes of radio telescope technologies have driven recent improvements in science capability. Broadly speaking, these new telescopes provide increases in sensitivity, field of view, accessible bandwidth, and often, combinations of these.

\subsubsection{Phased Array Feeds}

Phased array feeds (PAFs) are integrated collections of dual-polarization dipoles, mounted in the focal plane of a dish reflector, with the aim to sample the electric field intercepted by the antenna system completely. Beamforming techniques are used to form "digital" beams on the sky, and simultaneous beams can be used to image instantaneously a far larger field than would be otherwise possible through the use of a single receiver on the same reflector. Two prominent examples of this approach are the Aperture Tile In Focus (Apertif) upgrade to the Westerbork Synthesis Radio Telescope (WSRT) in the Netherlands [180] and the Mk-II PAFson the Australian Square Kilometre Array Pathfinder (ASKAP) [181] at the Murchison Radio-astronomy Observatory (MRO) in outback Western Australia.

These PAF-based systems deliver very large fields of view: for example, 9.5 and 30 square degrees with Apertif and ASKAP, respectively, with the difference between them largely driven by the antenna diameter ( 25 and $12 \mathrm{~m}$, respectively). Linking the individual antennas as an interferometric array ensures that an increase in field of view is achieved while retaining excellent angular resolution (typically, $\approx 5-30^{\prime \prime}$ ). Additionally, the digital PAF beamforming process is in principle highly flexible and offers the prospect for constraining the orthogonal components of polarization (these systems work on the basis of linear polarization, so " $X$ " and " $Y$ ") to have very similar beam shapes, and thereby providing extremely high polarimetric performance throughout the wide field of view.

PAFs are powerful for new magnetism surveys primarily because of their large field of view, which translates to a very high survey speed despite their typically lower sensitivity as compared to the new 
single-pixel receivers (described in Section 3.1.2). PAF systems also tend to deliver moderately large fractional bandwidth, with practical limits set by the desire to mosaic the same field of view with beams with frequency-dependent sizes, as well as realistic data management and processing constraints.

An example of the polarization performance of ASKAP is the mapping of the southern lobe of Centaurus A [182]. The excellent angular resolution, surface brightness sensitivity, and polarization performance together provided a new and highly detailed view of the magneto-ionic structure associated with this iconic radio galaxy.

\subsubsection{Wideband Single Pixel Feeds}

Modern single pixel receivers (here referred to as Wide-Band Single Pixel Feeds (WBSPFs)) are sensitive feeds across a very broad bandwidth. The newest of these receivers provides a performance comparable to the best traditional "octave" feeds (typical ratios of 1.85:1), but consistently across much broader bandwidths (ratios of 3:1 or greater). Prominent examples of the octave variety and relevant for new polarization surveys are MeerKAT, currently operating at L-band with an $800 \mathrm{MHz}$ bandwidth (880-1680 MHz)[183,184] and soon to be equipped with systems from $580-3700 \mathrm{MHz}$, and the Karl G. Jansky Very Large Array (VLA), which has continuous frequency coverage delivered by several contiguous octave feeds between 1 and $50 \mathrm{GHz}^{6}$ [185]. The Parkes radio telescope, as an SKA technology pathfinder, currently has an ultra-wide bandwidth receiver operating from $700 \mathrm{MHz}$ to $4 \mathrm{GHz}$ [186]. Parkes is also designing a comparable pair of systems to operate from 4 to $26 \mathrm{GHz}$.

A recent outcome demonstrating the value of the excellent spectropolarimetry that is now available with the VLA is the recognition of compressed upstream magnetised ISM associated with the Sagittarius spiral arm [187] through the detection of polarized extragalactic sources with RMs in excess of $10^{3} \mathrm{rad} \mathrm{m}^{-2}$. The ultra-wide band receiver at Parkes is already delivering broad bandwidth polarisation studies of pulsars (e.g., [188,189]), and there are also a number of ongoing projects focused on broadband continuum polarisation mapping.

\subsubsection{Aperture Arrays}

Aperture arrays have seen a dramatic resurgence in recent years. These are commonly used at low frequencies $(v<350 \mathrm{MHz})$. Aperture arrays are similar to PAFs, but are fixed on the ground rather than illuminated by a reflector. Key examples are the LOw Frequency ARray (LOFAR) [190] and the Murchison Widefield Array (MWA) [191,192].

Like PAFs, some aperture arrays can be used to observe multiple simultaneous fields of view across very wide areas, leading to flexible survey capabilities. Regardless of individual details, aperture arrays provide very wide fields of view and thus high survey speeds. They are very powerful for magnetism surveys because they provide extremely large fractional bandwidth, at a frequency range distinct from traditional work in the GHz regime.

Aperture arrays have proven to be productive for studying magnetism in the local and distant Universe, both with the MWA [193-195] and LOFAR [48,106,120,196]. Although radio sources tend to be substantially depolarized at low frequencies in dense environments, the detection rate is better in the outskirts of galaxy clusters or for field galaxies, and the precision with which Faraday rotation measures can be determined is excellent. Results from large-area extragalactic polarization surveys with both the MWA and LOFAR are discussed in Section 3.2.

\subsection{Upcoming Magnetism Projects}

In Figure 1, we demonstrate the continual and ongoing improvement of current and future surveys for the production of RM Grids. Each survey is represented by an indicative sky area and corresponding

6 https://science.nrao.edu/facilities/vla 
polarized source density. Additionally, the size of each marker reflects the angular resolution of the survey, and the colour indicates the nominal RM precision that can be reached. Diagonal dashed lines are intended to highlight the locus of "current" (lower) and "pathfinder" (upper) survey capability. The SKA1-MID survey stands out by having an exceptionally high source density over the full Southern sky, together with excellent angular resolution and RM precision. We now provide brief descriptions of the individual surveys.

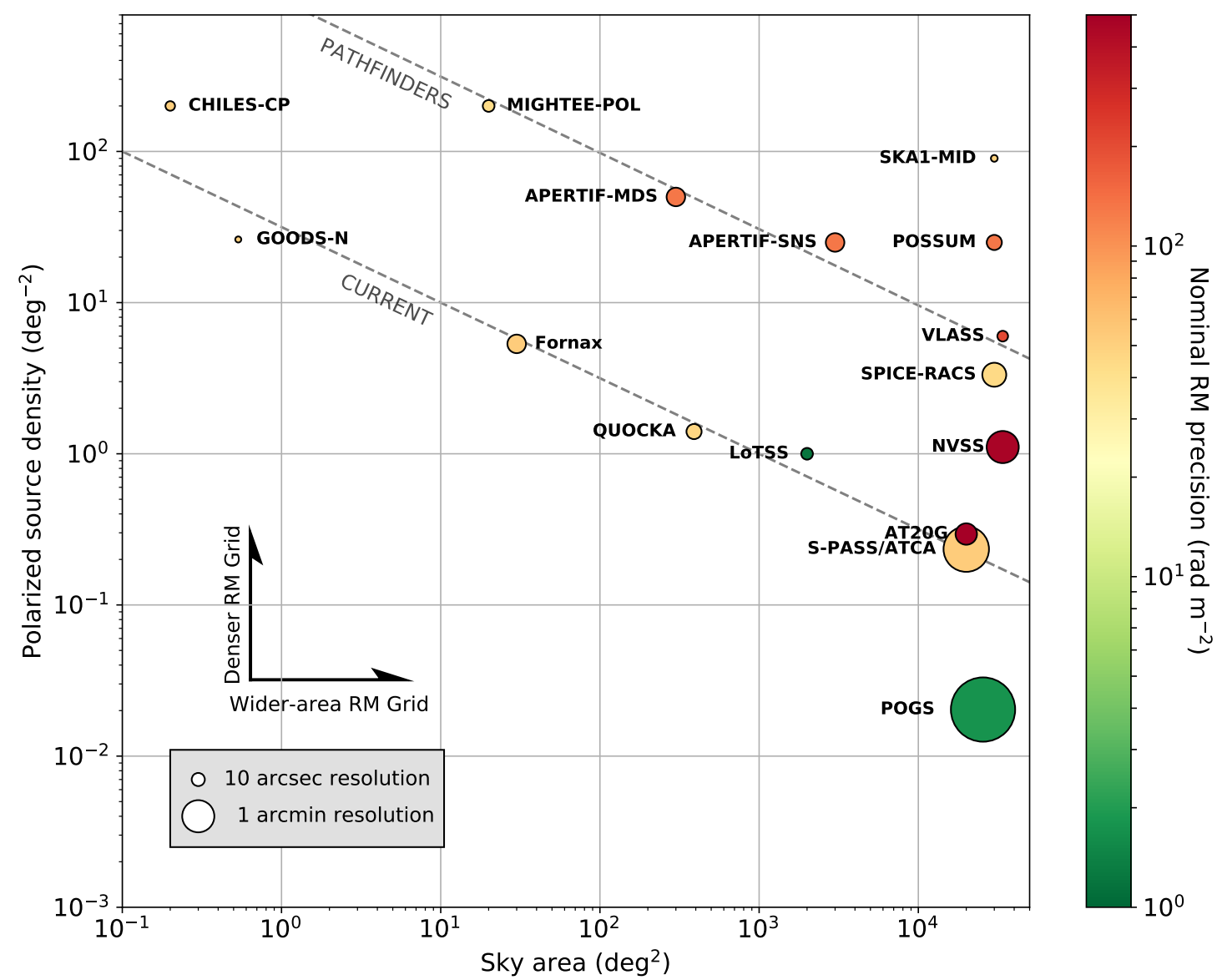

Figure 1. Illustration of the Rotation Measure (RM) grid survey strengths of various existing and future radio surveys. The size of each marker reflects the angular resolution of the survey, and the colour indicates the nominal RM precision that can be reached, where green indicates the capacity for measurements with lower RM uncertainties. Diagonal dashed lines are intended to highlight the locus of "current" (lower) and "pathfinder" (upper) survey capability.

ASKAP RACS. The Rapid ASKAP Continuum Survey (RACS; McConnell et al., in prep.) is the first all-Southern sky imaging survey completed using the full ASKAP array of 36 dishes. The primary goals of RACS are to establish an initial broadband sky model for calibration purposes and to provide a state-of-the-art continuum survey of the Southern sky. RACS is assembled from shallow, $15 \mathrm{~min}$ pointings, covering declinations $-90^{\circ}<\delta<+50^{\circ}$ with a target angular resolution of $12^{\prime \prime}$. Despite the short integrations, the RMS noise in Stokes $I$ is $\sim 300 \mu \mathrm{Jy} \mathrm{beam}^{-1}$, a significant improvement over previous surveys such as NVSS [197] and SUMSS [198]. A polarization component is being delivered in addition to the total intensity images and catalogue. This is called Spectra and Polarization In Cutouts of Extragalactic Sources from RACS (SPICE-RACS; Thomson et al., in prep). It will deliver RMs for a projected 100,000 radio sources, initially in the frequency range 744-1032 MHz, corresponding to a Faraday resolution of $44 \mathrm{rad} \mathrm{m}^{-2}$, at an angular resolution of $25^{\prime \prime}$. This frequency range will 
later be extended to include the $\sim 1150-1450 \mathrm{MHz}$ range, allowing for greater sensitivity to Faraday thick structures.

ASKAP POSSUM. The Polarization Sky Survey of the Universe's Magnetism (POSSUM) ${ }^{7}$ [199] is ASKAP's full-depth polarization survey. It is complementary to the Evolutionary Map of the Universe (EMU) survey [200]. POSSUM has long planned to provide frequency coverage from about 1130-1430 MHz, but may cover lower frequencies (similar to the initial RACS band as described above), depending on survey commensality and RFI avoidance. POSSUM will generate an RM Grid over a sky area of 30,000 square degrees, through imaging to a depth of about $20 \mu \mathrm{Jy}_{\text {beam }}{ }^{-1}$, resulting in up to about a million polarized sources. The angular resolution will be $\approx 10-15^{\prime \prime}$ and depending on final frequency coverage will deliver RM precision better than $4-13 \mathrm{rad} \mathrm{m}^{-2}$ for sources detected with signal-to-noise ratios in polarization of at least five. POSSUM will provide excellent surface brightness sensitivity to map extended emission in, for example, cluster relics and the lobes of radio galaxies.

ATCA QUOCKA. The QUOCKA (QU Observations at $\mathrm{Cm}$ wavelengths with $\mathrm{Km}$ baselines using ATCA) Survey ${ }^{8}$ (Heald et al., in prep.) will supplement our understanding of the polarized sources that will be detected with POSSUM, through targeted broadband observations of radio galaxies already detected in polarization during the ASKAP early science period. Using the ATCA, $\approx 550$ sources were observed from 1.1-3.1 and 4.5-8.5 GHz in several snapshots. These data will be used to develop a clear picture of the (de)polarization properties of radio galaxies and to inform the relatively narrower-band data that ASKAP will deliver. Because of the excellent polarization characteristics of ATCA, QUOCKA will also deliver a high-quality search for circular polarization probing the jet properties within the target sources.

VLASS. The VLA Sky Survey (VLASS) ${ }^{9}$ [185] is an effort to deliver a high-quality catalogue along with maps of radio sources over a bandwidth of $2-4 \mathrm{GHz}$. VLASS is conducted in an on-the-fly survey mode and includes a polarization component as a high priority goal. VLASS has some science aims similar to QUOCKA and adds well-resolved polarization images and a search for extreme RM sources, up to $\approx 16,000 \mathrm{rad} \mathrm{m}^{-2}$. In addition to RMs for compact sources, the high angular resolution $\left(\approx 2.5^{\prime \prime}\right.$ for total intensity, $\approx 5^{\prime \prime}$ for polarization) together with the broad bandwidth will allow a detailed study of the magneto-ionic structures in over 10,000 radio galaxies across the Universe. Figures 12 and 13 in [185] show the power of this high resolution for radio galaxy polarization studies.

MeerKAT. Several MeerKAT Large Survey Projects (LSPs) incorporate a polarization component, including in imaging mode: the MeerKAT International GHz Tiered Extragalactic Exploration (MIGHTEE) [201]; commensal polarimetry with the deep H I LADUMA (Looking at the Distant Universe with the MeerKAT Array) ${ }^{10}$ project [202]; and MHONGOOSE (MeerKAT H I Observations of Nearby Galactic Objects -Observing Southern Emitters) ${ }^{11}$ [203]. The MIGHTEE project team is planning complementary observations with the upgraded Giant Metrewave Radio Telescope (GMRT) to provide combined deep polarimetry of several square degrees with semi-contiguous frequency coverage from 300-2500 MHz. The deep field surveys will uniquely permit the study of magnetism in the very faint radio source population, expected to be dominated by distant star-forming galaxies. MHONGOOSE will probe magnetic fields in nearby galaxies through direct detection of diffuse synchrotron radiation, as well as the analysis of RMs associated with background radio galaxies. Additionally, the MeerTime project will regularly observe over 1000 radio pulsars using the MeerKAT array for a wide range of science cases, including probing the magneto-ionic ISM (e.g., [204,205]). Finally, the MeerKAT S-band Galactic plane survey, a MPIfR-SARAOcollaboration, will deliver polarization products tracing the Milky Way's magnetic field.

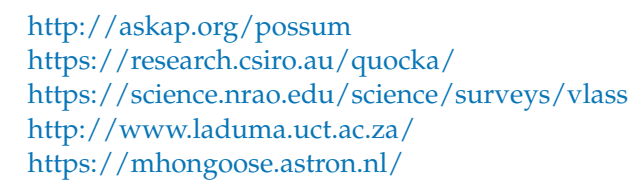


CHIME. The Canadian Hydrogen Intensity Mapping Experiment (CHIME) operates in the $400-800 \mathrm{MHz}$ band, with angular resolution $20-40^{\prime}$ and a field of view of $\sim 200^{\circ}$. It was designed for cosmological investigation of the history of the expansion of the Universe. To reach this goal, the Milky Way foreground will have to be subtracted to a precision of $10^{-5}$, implying that very precise imaging of total-intensity and polarized Galactic emission will be produced, covering the entire Northern sky. A subsidiary polarization survey with a $15 \mathrm{~m}$ telescope at the Dominion Radio Astrophysical Observatory (DRAO) will be performed over the same band. The $15 \mathrm{~m}$ survey will add very extended polarized structure, to which CHIME is insensitive and will provide polarization calibration. The polarization mapping is a joint project of the CHIME Collaboration and GMIMS ([206]; see below). Subsequent to its construction, CHIME was fitted with a second digital backend for investigation of FRBs and pulsars, including measurement of RMs [207].

LOFAR LoTSS. LOFAR is opening up the low-frequency $(v<200 \mathrm{MHz})$ polarized sky at high angular resolution $\left(6-10^{\prime \prime}\right)$ and finding that surveys for linearly polarized sources are more productive at these frequencies than had been anticipated some years ago $[194,196]$. Excellent progress is now being made through the ongoing LOFAR Two-metre Sky Survey (LoTSS) [208,209]. Studies of polarization in individual objects [47] and collections of sources [48,210] hold promise to trace the weak magnetic fields thought to thread the large-scale structure of the Universe (see also [42]). An RM Grid is being produced from LoTSS data, with around one RM measurement per square degree; leading to a full catalogue, which when complete should contain up to about 10,000 sources. The polarization maps of these sources will be at excellent angular resolution (6-20") and will have remarkably good $\mathrm{RM}$ resolution $\left(\mathcal{O}(1) \mathrm{rad} \mathrm{m}^{-2}\right)$. Complementary information will be provided by the WEAVE-LOFAR Survey [211] through observations in the optical band using the William Herschel Telescope Enhanced Area Velocity Explorer (WEAVE) spectrograph. The knowledge of the redshift of polarized radio sources will provide the means to evaluate the pathlength traversed by the signal along the line of sight, and thus allow inference of the magnetic field properties through statistical approaches and detailed studies of individual sources.

MWA POGS. In the Southern sky, the MWA is also developing an all-Southern sky view of extragalactic polarized sources. Using data from the GaLactic and Extragalactic All-Sky MWA (GLEAM) Survey [212-214], a catalogue of 500 sources has been developed (see [195]). This catalogue covers the entire sky south of declination $+30^{\circ}$, in the frequency range 169-231 MHz, for a nominal $\mathrm{RM}$ resolution of order $2.6 \mathrm{rad} \mathrm{m}^{-2}$. The POlarised GLEAM Survey (POGS) provides high-precision RMs for some 484 extragalactic radio sources and 33 known pulsars [215]. However, the low frequency and moderate resolution $\left(\approx 3^{\prime}\right)$ of POGS results in strong beam depolarisation due to fluctuations, including in the Galactic foreground, on scales below the PSF. Polarimetry with the Phase II MWA [192], which provides a factor $\sim 2$ improvement in resolution [216], will provide a big step forward in our understanding of the low-frequency polarised sky. This will not only come through reduced beam depolarisation and improved sensitivity, leading to an increased number of source detections in this comparatively unexplored sky area, but also through direct comparison of our Phase I and Phase II measurements, which will allow us to probe the scale size of Galactic magnetic field fluctuations.

GMIMS. The Global Magneto-Ionic Medium Survey (GMIMS) has set out to map the polarized emission from the entire sky, North and South, covering 300-1800 MHz with thousands of frequency channels, using large single-antenna telescopes [206]. GMIMS is mapping the sky in Faraday depth, making the first applications of RM synthesis to single-antenna data. The chosen frequency coverage provides a resolution in Faraday depth of $\sim 4 \mathrm{rad} \mathrm{m}^{-2}$ and an ability to detect Faraday depth structures up to $\sim 110 \mathrm{rad} \mathrm{m}^{-2}$ in extent. For technical reasons, the GMIMS band is broken into three bands, 300-800, 800-1300, and 1300-1800 MHz; all-sky surveys are being conducted in each band. To cover both hemispheres, the entire project comprises six component surveys. Three are completely observed: 300-900 MHz and 1300-1800 MHz with the Parkes $64 \mathrm{~m}$ Telescope, and 1270-1750 MHz with the DRAO $26 \mathrm{~m}$ Telescope. To date, only the range 300-480 MHz from Parkes has been published [132], but processing of the other two is well under way. The GMIMS project is designed to probe the magnetic 
field as a significant energy-carrying component of the ISM $[217,218]$ and to better understand ISM processes by including magnetic fields.

\section{Technical Considerations}

New techniques and methods are required to turn the raw data collected by SKA pathfinders and precursors, and eventually the SKA itself, into useful data products from which we can extract brand-new magnetism science results. In this section, we highlight several examples of new polarization-specific techniques and methods, following the order of data flow: from raw visibilities to high fidelity wide-field full-Stokes image cubes; from image cubes to enhanced polarization products; and from enhanced polarization products to magnetism science outcomes.

\subsection{Calibration and Widefield Imaging}

Most polarization surveys to be conducted with SKA pathfinders will consist of a mosaic of images formed from distinct telescope pointings, or from on-the-fly mapping, to cover the region of interest instead of a targeted on-axis approach. Therefore, to obtain high fidelity full-Stokes image cubes, we need to be able to extract reliable polarization information from sources away from the bore-sight; thus, the full-Stokes primary beam response needs to be taken into account. While on-axis instrumental polarization can be calibrated and corrected for using standard calibration procedures [219], off-axis instrumental polarization calibration is more complex $[194,195,220]$. Without proper off-axis instrumental polarization calibration, sources that are intrinsically unpolarized will artificially appear to be polarized, and sources that are in fact polarized will display polarization properties deviating from their true intrinsic values. This instrumental effect is a major limiting factor for robust RM determination across wide fields with current radio telescopes.

Correcting for these instrumental response terms is not only important for magnetism science, it is also highly relevant for EoR studies since other Stokes parameters can leak into Stokes I, and especially when the leakage signals have spectral dependencies, these can mimic or distort EoR signals [221]. It will be important for the SKA antenna design to start with good cross-polarization behaviour, but corrections will nonetheless be needed. The observed Stokes IQUV is the outer product of the direction-dependent Müller matrix characterising the primary beam response and the true Stokes IQUV (e.g., [222]). The correction is less complicated if the primary beam pattern does not rotate with respect to the sky throughout the observation and in that case can potentially be corrected in the image plane. In general, a full solution to the problem requires a full-Stokes Müller A-projection treatment, where the primary beam response in full-Stokes is projected out and corrected for in the imaging step [223]. In order to use this algorithm, well-characterized frequency-dependent beam models are required, using EM simulation results, holographic measurements, or a hybrid of these methods. Self-calibration is an alternative approach to correcting for wide-field instrumental polarization effects. For example, the work in [194] tested this approach on MWA data: as sources drift across the field of view, assuming they are unpolarized, one could map out a leakage surface and subtract it out from the observations. These techniques allow for high dynamic range, full-Stokes image cubes with minimal instrumental effects across wide fields of view.

Another important polarization-related calibration step is ionospheric RM correction. This is especially relevant at low frequencies (below $\sim 1 \mathrm{GHz}$ ) where the nominal uncertainty in Faraday rotation is less than the magnitude of ionospheric Faraday rotation (which can often take values up to at least $\sim 1-2 \mathrm{rad} \mathrm{m}^{-2}[178,193,224]$ and becomes more significant at solar maximum). In addition, over long integrations at low frequencies, the time-variable ionospheric RM can depolarize astrophysical signals and must therefore be corrected. These corrections are required in order to allow for magnetism projects that aim to detect very small astrophysical $R M$, or very small variations thereof. This includes probing the magnetic power spectrum on very small $(<\mathrm{pc})$ scales using pulsar proper motion and the associated RM time variabilities, the heliospheric RM, IGM magnetic fields at high redshifts, as well as the study of ionospheric properties (e.g., [225]). 
Ionospheric RM is usually approximated as the integral of the product of electron content and ionospheric magnetic fields in a thin shell approximation. Recently, the performance and the accuracy of publicly available global ionospheric maps have been rigorously compared using LOFAR pulsar RM observations [179]. While predictions using different geomagnetic fields mostly agree with each other, the accuracy of ionospheric RMs is dominated by the ionospheric total electron content maps: the JPLG [226] and UQRGmaps [227] are found to be superior. It is certainly beneficial to have local high-cadence TECmeasurements [228]. Alternatively, it has also been shown that one can use brightly polarized sources or the bright diffuse polarized synchrotron Galactic emission to track the ionospheric RM if one has polarization measurements of the field of interest at different epochs [193,229].

\subsection{Polarization-Specific Processing}

Once we have the fully-calibrated Stokes IQUV image cubes in hand, the development of a robust RM Grid catalogue that can be used to probe the science questions in Section 2 demands that we ask the following basic questions: Where are the polarized sources? What are their angular extents? What are their polarization properties, and how complex are their polarization behaviours as a function of wavelength $(\lambda)$ ? The first two questions can be addressed by source finding in polarization, while the latter two questions can be addressed by broadband polarization analysis tools such as RM synthesis [33,34], Stokes QU fitting [230,231], and classification of the degree of complexity exhibited in the Faraday dispersion functions (or "Faraday spectra"). All these tasks have to be accomplished with a reasonable amount of computing time and resources. Source finding in polarization is highly non-trivial [232,233]: noise in polarized intensity is non-Gaussian; individual sources can display polarized emission at more than one Faraday depth; and the peak in polarized intensity does not always coincide with that in Stokes $I$.

Performing source finding on Faraday depth cubes from RM synthesis might not be the most computationally effective approach because the sky is largely empty of compact polarized sources. One of the possibly more efficient methods to find polarized sources is the use of so-called Faraday moments [234], which are the mean, standard deviation, skewness, and excess kurtosis of the observed Stokes $Q, U$ and polarized intensity (PI) cubes. Another possibility is to analyse only subregions near total-intensity sources of interest [106]. In the former case, moment maps of the image cubes are produced, and then, normal source-finding software can be employed on these maps to find polarized sources. In both cases, RM synthesis will be performed on small subregions. This procedure greatly reduces the number of pixels for which one needs to perform RM synthesis, and hence decreases the required computing time, data storage, and network transport. The Faraday moment approach has been shown to be produce satisfactory completeness and can bypass the need for procedures that deal with non-Gaussian noise in polarized intensity. The more general source-finding problem is being actively tackled by various SKA pathfinder project teams, including ASKAP's POSSUM, which has a dedicated group that works on data challenges to assess the completeness and reliability of source finding strategies.

Further advances have been made in broadband polarization analysis tools in recent years. RM synthesis is a non-parametric approach to developing a model of source polarization properties from the observational data. Several implementations of the technique have been developed over the past fifteen years, mostly based on direct Fourier transforms (DFTs), but in at least one case through gridding the $\lambda^{2}$ data and performing a fast Fourier transform (FFT) ${ }^{12}$. Improved data quality (reduced errors and artefacts) should come about from combining the two-dimensional Fourier transform(s) used to form channel images from the visibilities and the one-dimensional transform embodied in RM synthesis to form a three-dimensional Fourier transform [235], a technique that holds interesting

12 https://github.com/mrbell/pyrmsynth 
promise despite high computational demands. Separately, standard RM synthesis has been accelerated through the use of GPUs [236].

Following RM synthesis, the resulting Faraday spectra can be deconvolved through a technique (RMCLEAN) similar to cleaning synthesis images [93]. This technique has been improved by refining the models generated by RMCLEAN in a maximum likelihood (ML) framework [237]. Currently ongoing research is seeking to optimise deconvolution in the channelised image plane for sources with low broadband signal-to-noise ratios.

Stokes QU fitting [230,231], on the other hand, is a parametric approach to describe broadband polarization data using models of the magnetized medium along the line of sight. Recent developments in this area include the FIRESTARTER algorithm [238], which takes into account the spectral indices of each of the fitted polarized components, and the use of convolutional neural networks (CNN) to classify Faraday depth spectra, to distinguish simple sightlines that exhibit only one RM component from more complex sightlines [239]. Interpretation of Faraday spectra in the case of turbulence can be complicated [126] and will require additional consideration.

Measuring polarization over broad bandwidths at low frequencies provides higher resolution in Faraday depth (see, e.g., Figure 1). Higher resolution in Faraday depth is vital for distinguishing between discrete Faraday screens and components, as well as subtle differences between RMs [48], implying tremendous potential value from polarization surveys with SKA1-LOW. At the same time, large RM values are becoming increasingly common within our Galaxy and in association with extragalactic sources such as FRBs [80]; understanding these extreme RM values can provide significant insight to the nature of the environment at and around the emission regions. However, large RM values at low frequencies undergo bandwidth depolarization. This depolarization will either cause sources with large RM to be undetected or incorrectly characterised in polarization at low frequencies. The amount of channel depolarization varies with channel width in $\lambda^{2}$, which can vary substantially over broad bandwidth, making it possible to generate signals that appear to show emission at more than one Faraday depth purely due to an instrumental effect. Recent work [240] developed the $\delta \lambda^{2}$-projection to model and correct the channel depolarization at low frequencies, which is analogous to the projection family of algorithms in interferometric imaging. Furthermore, this work makes it clear that many tools from interferometric imaging may be required for processing polarimetric signals over broad bandwidths.

\subsection{Getting to the Science: Analysis Tools}

Once we have enhanced polarization data products at hand, we need analysis tools that will enable us to deliver the actual cosmic magnetism science goals. For example, an information field theory (IFT)-based framework [241] has been used to reconstruct the Galactic RM sky from noisy measurements of discrete RMs towards background (extragalactic) polarized sources. The algorithm takes advantage of the fact that RMs induced by our Galaxy are spatially correlated, while extragalactic RMs and the observing noise should be spatially uncorrelated. Under this framework, the Galactic RM can be reconstructed, along with an uncertainty map. The original reconstruction [242] has been improved in recent years: first, by relying on fewer assumptions [243]; and also by folding in additional information about thermal gas along the line of sight [142]. These algorithms permit a statistical discrimination of the Galactic and extragalactic Faraday rotation. A further isolation of the extragalactic contribution can be accomplished with a Bayesian technique [244], particularly useful for enabling extragalactic magnetism studies. The assumption of an extragalactic uncorrelated term holds when dealing with the largest currently available RM catalogue (from NVSS data; [245]), characterized by a density of one source per square degree. However, this assumption will not be applicable any more with the catalogue obtained with the SKA1-MID RM Grid, which will be $\approx 100 \times$ denser, because lines of sight to adjacent sources will be near enough to pass through many of the same media, leading to a correlated extragalactic RM contribution. A complicating factor will be that individual resolved radio galaxies will themselves provide multiple samples of RM. 
There are also developments on new tools to identify and characterize structure in images, facilitating comparisons between maps of diffuse Galactic polarized emission and other tracers of the ISM. For example, the rolling Hough transform was first used to detect coherent linear features in H I maps [246] and is now being applied more broadly to seek correlations between the orientation of magnetic structures and different ISM phases [247]. Additionally, the polarization gradient method [125] can be used to constrain fundamental parameters of interstellar turbulence.

The development of the techniques described in this section has been required to keep up with the rapid capability advances coming from the new telescopes and instruments described in Section 3. As we progress through the pathfinder era and continue to refine these analysis techniques, we are increasingly preparing for the challenges of the SKA era.

\section{Survey Specifications}

A broadband polarisation survey covering a large sky area and with unprecedented sensitivity and resolution, as we expect to be enabled by the SKA, will allow us to address a broad range of scientific questions as reviewed in Section 2. Advancement in several characteristics of polarisation survey specifications is crucial in order to make substantial progress in measuring the polarised emission of cosmic sources and to gain new insight into the magnetic field of the Milky Way, extragalactic objects, and in the cosmic web. A substantial improvement in sensitivity will be required in order to detect more sources (raw sensitivity) and low surface brightness features (importance of short baselines). Excellent angular resolution (importance of long baselines) is important to disentangle source components, for example the lobes of distant radio galaxies, and to minimise the effects of beam depolarisation. Finally, a core aspect of an SKA polarisation survey is the frequency range, which establishes the RM range that can be studied, the degree of Faraday complexity that can be reliably recovered, and the associated precision in measured RMs. Observations at lower frequencies deliver better RM precision, but on the other hand, the fraction of depolarized sources increases towards lower frequencies. In this section, we discuss in detail the specifications of an optimal mid-frequency survey for mapping the polarized sky with the SKA [5], followed by additional considerations for ancillary survey activities.

\subsection{An SKA1-MID RM Grid Survey}

Frequency range. The radio astronomy community has long experience working within the L-band $(1-2 \mathrm{GHz})$, a frequency range that addresses a broad range of scientific questions from observations of radio continuum and $\mathrm{HI}$, for example. In the context of radio polarimetry, this is an excellent frequency range because it simultaneously provides reasonable RM precision (typically $\lesssim 10 \mathrm{rad} \mathrm{m}^{-2}$ for sources detected with sufficient signal-to-noise), while many sources that depolarise at lower frequencies are still polarised. Therefore, we plan to carry out a primary polarisation survey using SKA1-MID Band 2, from 950 to $1760 \mathrm{MHz}$ [5]. In comparison to POSSUM (originally $1130-1430 \mathrm{MHz}$, although subject to change as indicated in Section 3.2), this provides a larger span in $\lambda^{2}$-space by a factor of 2.7, so that SKA will deliver better RM precision by about the same factor. At the same time, broadband spectral structures including extended features in the Faraday spectrum can be recovered, allowing improved characterisation of Faraday thick sources. Based on the standard expressions [34], SKA1-MID Band 2 will provide a nominal RM precision $\Delta \mathrm{RM} \lesssim 5 \mathrm{rad} \mathrm{m}^{-2}$ for sources with signal-to-noise ratios $\mathrm{S} / \mathrm{N} \geq 5$ and partial sensitivity to resolved structures in Faraday space with a breadth up to around $108 \mathrm{rad} \mathrm{m}^{-2}$.

The rapidly changing RFI situation bears some brief discussion here. While both SKA sites are protected from ground-based interference, SKA1-MID Band 2 is nevertheless expected to be partially affected by aircraft and satellite communication. In particular, the range from $1025-1150 \mathrm{MHz}$ is populated by aircraft navigation, while $1217-1251 \mathrm{MHz}$ is used for Global Navigation Satellite 
System (GNSS) satellites ${ }^{13}$. Although we expect to be able to mitigate the direct impact of this RFI using standard practices, on the other hand, we also expect that the sidelobes resulting from RM synthesis will be substantially increased due to frequency gaps generated by RFI flagging: a naive analysis indicates that the innermost RM sidelobes will increase from $\sim 30 \%$ to $\sim 50 \%$, making reliable deconvolution techniques for RM spectra even more crucial.

Sensitivity. Meaningfully increasing the number of catalogued polarized sources compared to existing and SKA pathfinder surveys requires a sensitivity of $4 \mu \mathrm{Jy}$ beam $^{-1}$. Assuming that a flux density of five times the noise is required to measure the RM of a source confidently, the polarized flux density threshold for sources in the RM Grid catalogue would be approximately $20 \mu \mathrm{Jy} \mathrm{beam}^{-1}$, though we anticipate that we may be able to catalogue sources up to a factor of two fainter, depending on advances in data processing, source detection, and characterisation [248]. Based on studies of the faint polarized radio source population [249,250], we expect to be able to find $60-90$ polarised sources per square degree. Preliminary results from POSSUM indicate that ASKAP will be able to measure RMs over the full Southern sky with a density of 25-30 sources per square degree, in agreement with estimates developed in the same way, hence corroborating the expected density of polarised sources for the SKA polarisation survey.

Our current estimate for the observing time required per field in order to reach the target sensitivity at our desired resolution is $15 \mathrm{~min}$ [251]. We expect to require approximately 30,000 pointings to cover the observable sky and wish to observe at night; thus, the execution of the survey requires $\sim 2.5$ years including overhead (see also [5]). There are aims for developing a scanning mode for MeerKAT, similar to VLASS, which may significantly reduce the overhead.

Angular resolution. A crucial parameter for SKA polarimetry surveys is the nominal angular resolution. We aim to achieve improvements both over existing surveys, as well as ongoing SKA pathfinder polarisation surveys. Moreover, we aim to achieve a common angular resolution with total intensity continuum surveys in a comparable frequency band. We therefore aim for $2^{\prime \prime}$ resolution, matching the "legacy" reference Survey \#4 that is optimised for cross-identification with optical surveys, as described by [252] (their Table 1). This is significantly better than the expected angular resolution of POSSUM, around $10^{\prime \prime}$, and 2.5 times better than the significantly shallower VLASS's resolution at $3 \mathrm{GHz}$. This desired improvement in angular resolution is crucial to isolate distinct RM components across a large fraction of resolved sources in the image plane directly. For example, many double radio sources will be clearly separated into two components at $2^{\prime \prime}$ resolution, and more subtle variations across sources will be ubiquitous. The angular resolution is also essential to obtain higher quality RMs as elements of an RM Grid (see [253] for a more detailed assessment of factors crucial for optimising the quality of the SKA RM Grid).

According to a recent simulation [254], the confusion limit in Stokes $Q$ and $U$ for this survey is $0.4 \mathrm{nJy}_{\text {beam }}{ }^{-1}$, well below the expected sensitivity level of $4 \mu \mathrm{Jy}$ beam $^{-1}$. This indicates that, in principle, substantially deeper targeted observations in polarization with the same observing setup could be performed without being limited by confusion noise.

$(u, v)$ coverage. A crucial aspect of the expected improvement in SKA polarization survey quality is the far more complete instantaneous $(u, v)$ coverage in SKA1-MID observations as compared to current facilities (expected rmsof near-in PSF sidelobes is well below 1\%; [255]). Even if difficult to quantify in advance over the full survey area, the larger number of baselines will allow a significantly better image reconstruction for complex extended sources and their polarised emission. Although SKA1-MID will itself provide exquisite sensitivity to emission on angular scales up to $\approx 0.5-1^{\circ}$, as an interferometer, it will still be subject to missing short spacings, and we anticipate that supplementary single-dish observations will be required for Milky Way research.

13 See, for example: https://science.nrao.edu/facilities/vla/docs/manuals/obsguide/rfi 
Sky coverage. We advocate a survey that covers the entire accessible sky from the SKA1-MID site, i.e., 30,000 square degrees. There are a few primary reasons for this preference. First, a comprehensive understanding of the entire visible Milky Way, through background-source probes and mapping of individual Galactic sources and diffuse emission at all Galactic latitudes, is essential not only for the study of the Galaxy itself, but also to ensure a high-quality foreground model for interpreting extragalactic objects and supporting complementary research programs (including FRBs and cosmological studies). Moreover, research projects that rely on large numbers of individual sources are best served with wide-area, rather than deep, but narrow-area, survey observations. For magnetism studies in particular, robust statistical analysis differentiating galaxy sub-classifications requires large numbers of sources, and probes of redshift evolution also require very large catalogues for unambiguous results. Finally, support for transient studies (counterpart identification and multi-messenger follow-up) requires an all-sky foundation. On the basis of such an all-sky SKA1-MID Band 2 survey, we expect to catalogue up to about three million polarized sources [254] to form the SKA1-MID RM Grid.

Commensality. As noted above, the SKA1-MID survey described here has the same specifications as the "legacy" reference continuum Survey \#4 described by [252]. However, the relative priority of the reference continuum surveys currently places more emphasis on narrower, more sensitive survey projects. This calls for a more active discussion about survey commensality between science teams, especially those with plans for large-area SKA1-MID surveys, possibly including H I mapping.

Data products and analysis. We aim to provide data cubes with sufficient frequency resolution so that we retain sensitivity to the expected RMs. The native spectral resolution of SKA1-MID is $13.4 \mathrm{kHz}$, which would allow full-sensitivity recovery of emission at RMs up to a few times $10^{5} \mathrm{rad} \mathrm{m}^{-2}$, expected in some environments as described in Section 2.1.5. However, by retaining this frequency resolution over the full bandwidth, individual cubes covering a sky area of only one square degree would each require 7 TB of disk storage. Moderate RMs of up to about $10^{4} \mathrm{rad} \mathrm{m}^{-2}$ (more than sufficient for the vast majority of magnetism science cases) would be retained with a more reasonable $1 \mathrm{MHz}$ spectral resolution, with which each cube would only require about $95 \mathrm{~GB}$. At this frequency resolution, IQUVcubes for all pointings for the full RM Grid survey would amount to approximately 5 PB. This data storage aspect is discussed further in Section 6.2.

RM synthesis and subsequently $Q U$ fitting will be used to identify and characterise the RM spectrum for each detected source. Through active research programs on SKA pathfinder telescopes, the community is currently optimising the procedures for this analysis stage to ensure efficient extraction of reliable source characteristics.

\subsection{Additional Considerations}

Beyond the specific requirements for our planned SKA1-MID Band 2 RM Grid survey, we note some additional aspects that bear attention in light of recent progress and results. These may form the basis of updates to our plans as we approach the SKA era and/or ancillary ideas for additional SKA Key Science Projects.

Dedicated efforts are now under way with SKA pathfinder telescopes (Section 3, especially headline surveys such as POSSUM and MIGHTEE). These are developing new knowledge and expertise, and as they push into new parameter space, they will allow us to learn how to develop and utilise these deeper polarization data products properly. On the basis of the new knowledge that will be gathered between now and the establishment of specific plans to execute SKA surveys, we should be alert to opportunities to improve the survey strategy and the associated plans for data analysis.

As outlined above, a key aspect of polarization surveys is the total frequency coverage, and in particular, the total span in $\lambda^{2}$-space is crucial for maximising the RM precision that can be expected. Moreover, the evolution of the polarized source population over a wide frequency range, along with broadband depolarization and re-polarization behaviours of individual sources are also of current interest. In the long term, the magnetism community may find that there is strong interest in a 
further increase in bandwidth by combining surveys across an even larger contiguous frequency range, and in particular SKA1-MID Band 1 (350-1050 MHz) to complement the primary Band 2 survey described here.

As noted above, the confusion level for polarization is expected to be far below the noise level that we aim to achieve for the SKA RM Grid survey. This leaves considerable opportunity for deep imaging surveys probing into the faint polarized source population, and probing the evolution of magnetism to high redshift, for example. A deep survey to complement the all-sky RM Grid survey is likely to be of particular interest.

Finally, we note the exciting new polarization results that are presently emerging from LOFAR and its all-sky imaging survey LoTSS [48,208]. Recently, exploitation of total intensity and polarimetric LOFAR observations demonstrated that these frequencies are powerful to address the study of magnetisation in as-yet unexplored systems. This advance is due to the capability for revealing low surface brightness radio sources (e.g., $[43,256])$ and dispersion in Faraday rotation down to $1 \mathrm{rad} \mathrm{m}^{-2}$ or less, likely associated with low-density and weakly magnetized environments, while being completely blind to dense and highly magnetized environments (e.g., $[48,210]$ ). These results highlight the importance of SKA1-LOW observations in combination with SKA1-MID, for a comprehensive study of cosmic magnetism in different magnetic field strength and thermal gas density regimes. It is clear that there is tremendous opportunity for SKA1-LOW to continue to probe this opening window to much higher sensitivity and over a broader frequency range than is currently being pursued with LOFAR. Plans for such a survey, which would be highly complementary to the MID RM Grid survey, will be developed based on the experience developed from LOFAR and with a view toward commensality with other SKA science areas.

\section{Looking Forward}

\subsection{SKA Data Challenges}

The SKA Organisation has recently commenced a program of "SKA data challenges", which aims to familiarise the user community with standard SKA data products and to help working groups develop and provide input into the associated processing and analysis pipelines [257]. The first data challenge, issued in November 2018 and concluded in July 2019, consisted of simulated SKA images at $0.56,1.4$, and $9.2 \mathrm{GHz}$, but only in total intensity and with no spectral index information. As such, this initial simulated dataset was not suitable for exploring the various challenges associated with polarimetry and magnetism science.

Future SKA data challenges will contain polarisation information, hopefully at successively greater levels of complexity. The first step will be to include a spectral index, polarised fraction, polarisation angle, and RM for each total intensity component, which will allow the Magnetism Science Working Group explore basic polarisation pipelines that employ polarisation source finding, $\mathrm{RM}$ synthesis, or $Q U$ fitting. The community has already begun to undertake simple polarisation source challenges in this vein [258], which highlight the difficulties that current algorithms experience even with relatively simple situations. A concerted effort will be needed to improve these approaches for SKA.

An initial round of enhancements might include depolarising effects due to multiple RMs within the synthesized beam, Faraday thick structures, spectral index effects, non-zero synchrotron optical depths, and bandwidth depolarisation within each spectral channel. Further effects will include spatially-extended sources (in which the polarised morphology and number of polarised components do not match their total intensity counterparts), the presence of diffuse polarised foregrounds $[193,259,260]$, polarisation leakage (both on- and off-axis), ionospheric Faraday rotation, and averaging of this leakage due to sky rotation or mosaicing. It is important to appreciate that simulating every conceivable polarisation property of an SKA observation is potentially an even bigger 
challenge than recovering them and that it is unlikely that any data challenge will fully capture all aspects of the polarised sky.

\subsection{SKA Regional Centres}

Data products for the SKA will be made available to users via a network of SKA Regional Centres (SRCs) [261], which will provide a range of services including archiving, user support, and custom processing and re-processing. There will be a core set of products offered by all SRCs, but also some unique services that perhaps only a subset of SRCs will provide. The aim is that this will be transparent to SKA users, who need not know in which SRC their data are located and will be able to access their data regardless of where they are based or affiliated.

Magnetism science with SKA will have unique requirements with regard to the data products produced and hosted by SRCs, centred on two main themes. First, magnetism experiments almost always require information in the Stokes parameters $Q$ and $U$, and often also in $I$ and $V$. There will always be leakage between Stokes parameters (see Section 4.1), which will be a complex function of frequency, location on the sky, and offset from the pointing centre. Leakage effects will need to be corrected for in calibration and in post-processing, and residual leakage will need to be appropriately characterised so that the user can set thresholds for what constitutes a valid measurement.

Second, the main data-taking mode for magnetism projects will be spectropolarimetry, which has distinct requirements from both continuum (total intensity) and spectral line observations. For most continuum experiments, the behaviour of intensity as a function of frequency will be captured in the form of Taylor terms, which describe the mean intensity, spectral index, spectral curvature, and higher order terms across the band. This is unsuitable for Faraday rotation and other polarimetry experiments, where the behaviour in Stokes $Q$ and $U$ can be highly complicated and oscillatory as a function of frequency and which cannot be efficiently described as a small set of Taylor terms. Rather, full-Stokes frequency cubes will need to be produced, stored, and analysed, so that the behaviour of $Q$ and $U$ as a function of frequency can be studied according to the user's needs. For most spectral line experiments, image cubes will be produced at very high spectral resolution, often over a relatively narrow bandwidth. For polarimetry with SKA1-MID, the spectral resolution needed will be modest ( $\approx 1 \mathrm{MHz}$ ), but the total bandwidth will be broad ( $\gg 100 \mathrm{MHz}$ ). For SKA1-LOW, the spectral resolution needs to be higher, but the spectral bandwidth is also narrower. In both cases, the sidelobe pattern, resolution, and field of view will vary significantly across the band, removing much of the efficiency or commonality in processing that might be employed for a spectral line cube. Furthermore, the dynamic range for many spectral line experiments is fairly low, meaning that deconvolution per channel is relatively unimportant; this will often not be the case for spectropolarimetry.

Some of the corresponding polarisation products that the SRC network will need to make available include:

- A catalogue of polarisation and Faraday rotation properties for each detected polarised component, using a standard format ${ }^{14}$;

- A cross-listing of detected polarised components matched to their component or source counterparts from total intensity;

- "Coarse" image cubes of IQUV, covering the entire survey area at modest $(\sim 1 \mathrm{MHz})$ spectral resolution;

- Possible "fine" image cubes of $I Q U V$, covering some subset of the sky at high spectral resolution (up to the maximum spectral resolution that will be available over the full bandwidth, $13.4 \mathrm{kHz}[251])$;

- Cubes of Faraday depth, either cut-outs around detected sources or covering the entire sky;

- Images of the peak Faraday depth and associated polarized intensity.

14 See https://github.com/Cameron-Van-Eck/RMTable for a proposed format. 
The main polarisation catalogue will need to contain a large number of parameters, including: the component's image coordinates; flux, polarised fraction, and position angle at a fiducial wavelength; peak Faraday depth; and uncertainties on all these parameters. Additionally, the catalogue needs to characterise the presence of multiple features in the Faraday depth spectrum.

Multiple developments are taking place worldwide to establish the first elements of the SRC network. For example, a prototype SRC has been built and is running at the Shanghai Astronomical Observatory, Chinese Academy of Sciences [262], which is open to the community. Similarly, Australia has established a prototype SRC (AusSRC), which is working with the SKA precursor telescopes ASKAP and MWA, providing support for these teams as they develop methods of dealing with large-scale data. In Canada, the Canadian Initiative for Radio Astronomy Data Analysis (CIRADA) 15 is producing enhanced and science-ready data products for polarization from POSSUM, VLASS, and CHIME, and is also acting as a pilot study for an anticipated Canadian SRC. In South Africa, the Inter-University Institute for Data Intensive Astronomy ${ }^{16}$, in partnership with several South African institutions, has established a data intensive research cloud ${ }^{17}$ prototyping SRC technologies, as well as processing and analytics tools for SKA pathfinder Large Survey Programs on MeerKAT and the upgraded GMRT.

\section{Summary}

In this contribution, we revisited the science goals and primary survey plans of the SKA Cosmic Magnetism Science Working Group, originally detailed about five years ago [5], in light of the rapid observational progress that is taking place within the field. It is clearly the case that SKA pathfinder telescopes and associated survey efforts are making substantial headway toward the capability that will be required to make the most of the SKA.

In the next few years, we will see the emergence of an all-sky RM Grid at intermediate source density from ASKAP POSSUM and VLASS, taking us partway toward the transformational capability that will be provided by the SKA RM Grid; and exquisite deep polarization images from MeerKAT MIGHTEE, which will inspire even deeper efforts with the SKA. The magnetism community stands at the threshold of a rich observational opportunity.

Author Contributions: Writing, original draft preparation, G.H., S.A.M., V.V., T.A., A.D.-S., B.M.G., and M.H.; writing, review and editing, I.A., A.B. (Aritra Basu), R.B., M.B., A.B. (Annalisa Bonafede), T.L.B., A.B. (Andrea Bracco), E.C., L.F., J.M.G., F.G., J.A.G., J.H., M.H., C.H., M.J.-H., R.K., T.L., B.N.-W., S.P.O'., M.P., F.P., L.P., M.R., C.J.R., T.R., L.R., C.S., J.M.S., X.S., S.S., A.R.T., A.T. C.L.V.E., F.V., and J.L.W.; Conceptualization, the SKA Magnetism Science Working Group. All authors read and agreed to the published version of the manuscript.

Funding: The Dunlap Institute is funded through an endowment established by the David Dunlap family and the University of Toronto. B.M.G. and J.L.W. acknowledge the support of the Natural Sciences and Engineering Research Council of Canada (NSERC) through Grant RGPIN-2015-05948 and of the Canada Research Chairs program. I.A. acknowledges support by a Ramón y Cajal grant (RYC-2013-14511) of the "Ministerio de Ciencia, Innovación, y Universidades (MICIU)" of Spain, financial support from MCIU through Grant AYA2016-80889-P, and through the "Center of Excellence Severo Ochoa" award for the Instituto de Astrofísica de Andalucía-CSIC (SEV-2017-0709). A.B[asu]. acknowledges financial support by the German Federal Ministry of Education and Research (BMBF) under Grant 05A17PB1 (Verbundprojekt D-MeerKAT). A.B. (Andrea Bracco) acknowledges the support of the European Union's Horizon 2020 research and innovation program under the Marie Skłodowska-Curie Grant Agreement No. 843008 (MUSICA). M.H. acknowledges funding from the European Research Council (ERC) under the European Union's Horizon 2020 research and innovation programme (Grant Agreement No. 772663). M.P. acknowledges funding from the INAF PRIN-SKA 2017 program 1.05.01.88.04. F.P. acknowledges support from the Spanish Ministerio de Ciencia, Innovación y Universidades (MICINN) under Grant Numbers ESP2015-65597-C4-4-R and ESP2017-86852-C4-2-R. M.R. acknowledges the "Departments of Excellence 2018-2022" grant awarded by the Italian Ministry of Education, University and Research (MIUR) L. 232/2016; Research grant TAsP (Theoretical Astroparticle Physics) funded INFN ; Research grant "Deciphering the high-energy sky via cross correlation" funded by the agreement ASI-INAFn. 2017-14-H.0; research grant

\footnotetext{
5 http://www.cirada.org

6 http://www.idia.ac.za

http://www.ilifu.ac.za
} 
"From Darklight to Dark Matter: understanding the galaxy/matter connection to measure the Universe" No. 20179P3PKJ funded by MIUR. C.J.R. acknowledges financial support from the ERC Starting Grant "DRANOEL", Number 714245. Partial support for L.R. comes from U.S. National Science Foundation Grant AST17-14205 to the University of Minnesota. J.M.S. acknowledges the support of the Natural Sciences and Engineering Research Council of Canada (NSERC), 2019-04848. X.S. is supported by the National Key R\&D Program of China (2018YFA0404602). S.S. acknowledges support from the Science and Engineering Research Board (SERB) of the Department of Science and Technology (DST), Govt. of India, through Research Grant ECR/2017/001535. F.V. acknowledges financial support of the H2020 initiative through the ERC Starting Grant MAGCOW(714196).

Acknowledgments: G.H. thanks Phil Edwards for carefully reading the manuscript and providing useful feedback.

Conflicts of Interest: The authors declare no conflict of interest.

\section{References}

1. Gaensler, B.M.; Beck, R.; Feretti, L. The origin and evolution of cosmic magnetism. Neww Astron. Rev. 2004, 48, 1003-1012. [CrossRef]

2. Feretti, L.; Burigana, C.; Enßlin, T.A. Diffuse radio emission from the intracluster medium. New Astron. Rev. 2004, 48, 1137-1144. [CrossRef]

3. Feretti, L.; Johnston-Hollitt, M. Magnetic fields in clusters of galaxies. New Astron. Rev. 2004, 48, 1145-1150. [CrossRef]

4. Beck, R.; Gaensler, B.M. Observations of magnetic fields in the Milky Way and in nearby galaxies with a Square Kilometre Array. New Astron. Rev. 2004, 48, 1289-1304. [CrossRef]

5. Johnston-Hollitt, M.; Govoni, F.; Beck, R.; Dehghan, S.; Pratley, L.; Akahori, T.; Heald, G.; Agudo, I.; Bonafede, A.; Carretti, E.; Clarke, T.; et al. Using SKA Rotation Measures to Reveal the Mysteries of the Magnetised Universe. In Advancing Astrophysics with the Square Kilometre Array (AASKA14); Sissa Medialab: Trieste, Italy, 2015; p. 92.

6. Agudo, I.; Boettcher, M.; Falcke, H.D.E.; Georganopoulos, M.; Ghisellini, G.; Giovannini, G.; Giroletti, M.; Gurvits, L.; Gómez, J.L.; Laing, R.; et al. Studies of Relativistic Jets in Active Galactic Nuclei with SKA. In In Advancing Astrophysics with the Square Kilometre Array (AASKA14); Sissa Medialab: Trieste, Italy, $2015 ;$ p. 93.

7. Gaensler, B.; Agudo, I.; Akahori, T.; Banfield, J.; Beck, R.; Carretti, E.; Farnes, J.; Haverkorn, M.; Heald, G.; Jones, D.; et al. Broadband Polarimetry with the Square Kilometre Array: A Unique Astrophysical Probe. In Advancing Astrophysics with the Square Kilometre Array (AASKA14); Sissa Medialab: Trieste, Italy, 2015; p. 103.

8. Laing, R. Kinematics and Dynamics of kiloparsec-scale Jets in Radio Galaxies with SKA. In Advancing Astrophysics with the Square Kilometre Array (AASKA14); Sissa Medialab: Trieste, Italy, 2015; p. 107.

9. Peng, B.; Chen, R.R.; Strom, R. Giant radio galaxies as probes of the ambient WHIM in the era of the SKA. In Advancing Astrophysics with the Square Kilometre Array (AASKA14); Sissa Medialab: Trieste, Italy, 2015; p. 109.

10. Beck, R.; Bomans, D.; Colafrancesco, S.; Dettmar, R.J.; Ferrière, K.; Fletcher, A.; Heald, G.; Heesen, V.; Horellou, C.; Krause, M.; et al. Structure, dynamical impact and origin of magnetic fields in nearby galaxies in the SKA era. In Advancing Astrophysics with the Square Kilometre Array (AASKA14); Sissa Medialab: Trieste, Italy, 2015; p. 94.

11. Heald, G.; Beck, R.; de Blok, W.J.G.; Dettmar, R.J.; Fletcher, A.; Gaensler, B.; Haverkorn, M.; Heesen, V.; Horellou, C.; Krause, M.; et al. Magnetic Field Tomography in Nearby Galaxies with the Square Kilometre Array. In Advancing Astrophysics with the Square Kilometre Array (AASKA14); Sissa Medialab: Trieste, Italy, 2015; p. 106.

12. Bonafede, A.; Vazza, F.; Brüggen, M.; Akahori, T.; Carretti, E.; Colafrancesco, S.; Feretti, L.; Ferrari, C.; Giovannini, G.; Govoni, F.; et al. Unravelling the origin of large-scale magnetic fields in galaxy clusters and beyond through Faraday Rotation Measures with the SKA. In Advancing Astrophysics with the Square Kilometre Array (AASKA14); Sissa Medialab: Trieste, Italy, 2015; p. 95.

13. Johnston-Hollitt, M.; Dehghan, S.; Pratley, L. Using Tailed Radio Galaxies to Probe the Environment and Magnetic Field of Galaxy Clusters in the SKA Era. In Advancing Astrophysics with the Square Kilometre Array (AASKA14); Sissa Medialab: Trieste, Italy, 2015; p. 101. 
14. Govoni, F.; Murgia, M.; Xu, H.; Li, H.; Norman, M.; Feretti, L.; Giovannini, G.; Vacca, V.; Bernardi, G.; Bonafede, A.; et al. Cluster magnetic fields through the study of polarized radio halos in the SKA era. In Advancing Astrophysics with the Square Kilometre Array (AASKA14); Sissa Medialab: Trieste, Italy, 2015; p. 105.

15. Haverkorn, M.; Akahori, T.; Carretti, E.; Ferrière, K.; Frick, P.; Gaensler, B.; Heald, G.; Johnston-Hollitt, M.; Jones, D.; Landecker, T.; et al. Measuring magnetism in the Milky Way with the Square Kilometre Array. In Advancing Astrophysics with the Square Kilometre Array (AASKA14); Sissa Medialab: Trieste, Italy, 2015; p. 96.

16. Han, J.L.; van Straten, W.; Lazio, T.J.W.; Deller, A.; Sobey, C.; Xu, J.; Schnitzeler, D.; Imai, H.; Chatterjee, S.; Macquart, J.P.; et al. Three-dimensional Tomography of the Galactic and Extragalactic Magnetoionic Medium with the SKA. In Advancing Astrophysics with the Square Kilometre Array (AASKA14); Sissa Medialab: Trieste, Italy, 2015; p. 41.

17. Dickinson, C.; Beck, R.; Crocker, R.; Crutcher, R.M.; Davies, R.D.; Ferrière, K.; Fuller, G.; Jaffe, T.R.; Jones, D.; Leahy, P.; et al. SKA studies of in situ synchrotron radiation from molecular clouds. In Advancing Astrophysics with the Square Kilometre Array (AASKA14); Sissa Medialab: Trieste, Italy, 2015; p. 102.

18. Robishaw, T.; Green, J.; Surcis, G.; Vlemmings, W.H.T.; Richards, A.M.S.; Etoka, S.; Bourke, T.; Fish, V.; Gray, M.D.; Imai, H.; et al Measuring Magnetic Fields Near and Far with the SKA via the Zeeman Effect. In Advancing Astrophysics with the Square Kilometre Array (AASKA14); Sissa Medialab: Trieste, Italy, 2015; p. 110.

19. Vazza, F.; Ferrari, C.; Bonafede, A.; Brüggen, M.; Gheller, C.; Braun, R.; Brown, S. Filaments of the radio cosmic web: opportunities and challenges for SKA. In Advancing Astrophysics with the Square Kilometre Array (AASKA14); Sissa Medialab: Trieste, Italy, 2015; p. 97.

20. Giovannini, G.; Bonafede, A.; Brown, S.; Feretti, L.; Ferrari, C.; Gitti, M.; Govoni, F.; Murgia, M.; Vacca, V. Mega-parsec scale magnetic fields in low density regions in the SKA era: filaments connecting galaxy clusters and groups. In Advancing Astrophysics with the Square Kilometre Array (AASKA14); Sissa Medialab: Trieste, Italy, 2015; p. 104.

21. Colafrancesco, S.; Regis, M.; Marchegiani, P.; Beck, G.; Beck, R.; Zechlin, H.; Lobanov, A.; Horns, D. Probing the nature of Dark Matter with the SKA. In Advancing Astrophysics with the Square Kilometre Array (AASKA14); Sissa Medialab: Trieste, Italy, 2015; p. 100.

22. Stil, J.; Keller, B. Stacking for Cosmic Magnetism with SKA Surveys. In Advancing Astrophysics with the Square Kilometre Array (AASKA14); Sissa Medialab: Trieste, Italy, 2015; p. 112.

23. Taylor, R.; Agudo, I.; Akahori, T.; Beck, R.; Gaensler, B.; Heald, G.; Johnston-Hollitt, M.; Langer, M.; Rudnick, L.; Scaife, A.; Schleicher, D.; Stil, J.; Ryu, D. SKA Deep Polarization and Cosmic Magnetism. In Advancing Astrophysics with the Square Kilometre Array (AASKA14); Sissa Medialab: Trieste, Italy, 2015; p. 113.

24. Vacca, V.; Oppermann, N.; Ensslin, T.A.; Selig, M.; Junklewitz, H.; Greiner, M.; Jasche, J.; Hales, C.A.; Reneicke, M.; Carretti, E.; et al. Statistical methods for the analysis of rotation measure grids in large scale structures in the SKA era. In Advancing Astrophysics with the Square Kilometre Array (AASKA14); Sissa Medialab: Trieste, Italy, 2015; p. 114.

25. Han, J.L. Observing Interstellar and Intergalactic Magnetic Fields. Aunn. Rew. 2017, 55, 111-157. [CrossRef]

26. Akahori, T.; Nakanishi, H.; Sofue, Y.; Fujita, Y.; Ichiki, K.; Ideguchi, S.; Kameya, O.; Kudoh, T.; Kudoh, Y.; Machida, M.; et al. Cosmic magnetism in centimetre- and meter-wavelength radio astronomy. Publ. Astron. Soc. Jpn. 2018, 70, R2. [CrossRef]

27. Heald, G., Synchrotron Radiation and Faraday Rotation. In Magnetic Fields in Diffuse Media; Lazarian, A., de Gouveia Dal Pino, E.M., Melioli, C., Eds.; Astrophysics and Space Science Library; Springer: Basel, Switzerland, 2015; Voume 407, p. 41. [CrossRef]

28. Crutcher, R.M.; Kemball, A.J. Review of Zeeman Effect Observations of Regions of Star Formation K Zeeman Effect, Magnetic Fields, Star formation, Masers, Molecular clouds. Front. Astron. Space Sci. 2019. [CrossRef]

29. Robishaw, T. Zeeman Splitting. Galaxies 2020, 8, in preparation.

30. Carilli, C.L.; Rawlings, S. Motivation, key science projects, standards and assumptions. New Astron. Rev. 2004, 48, 979-984. [CrossRef]

31. O'Sullivan, S.P.; McClure-Griffiths, N.M.; Feain, I.J.; Gaensler, B.M.; Sault, R.J. Broad-band radio circular polarization spectrum of the relativistic jet in PKS B2126-158. Mon. Not. R. Astron. Soc. 2013, 435, 311-319. [CrossRef]

32. Gruzinov, A.; Levin, Y. Conversion Measure of Faraday Rotation-Conversion with Application to Fast Radio Bursts. Astrophys. J. 2019, 876, 74. [CrossRef] 
33. Burn, B.J. On the depolarization of discrete radio sources by Faraday dispersion. Mon. Not. R. Astron. Soc. 1966, 133, 67. [CrossRef]

34. Brentjens, M.A.; de Bruyn, A.G. Faraday rotation measure synthesis. Astron. Astrophys. 2005, 441, 1217-1228. [CrossRef]

35. Haverkorn, M.; Machida, M.; Akahori, T. Workshop Summary "The Power of Faraday Tomography". Galaxies 2019, 7, 26. [CrossRef]

36. Han, J.L.; Manchester, R.N.; van Straten, W.; Demorest, P. Pulsar Rotation Measures and Large-scale Magnetic Field Reversals in the Galactic Disk. Astrophys. J. Suppl. Ser. 2018, 234, 11. [CrossRef]

37. Cen, R.; Ostriker, J.P. Where Are the Baryons? Astrophys. J. 1999, 514, 1-6. [CrossRef]

38. Davé, R.; Cen, R.; Ostriker, J.P.; Bryan, G.L.; Hernquist, L.; Katz, N.; Weinberg, D.H.; Norman, M.L.; O'Shea, B. Baryons in the Warm-Hot Intergalactic Medium. Astrophys. J. 2001, 552, 473-483. [CrossRef]

39. Brown, S.; Vernstrom, T.; Carretti, E.; Dolag, K.; Gaensler, B.M.; Staveley-Smith, L.; Bernardi, G.; Haverkorn, M.; Kesteven, M.; Poppi, S. Limiting magnetic fields in the cosmic web with diffuse radio emission. Mon. Not. R. Astron. Soc. 2017, 468, 4246-4253. [CrossRef]

40. Vernstrom, T.; Gaensler, B.M.; Brown, S.; Lenc, E.; Norris, R.P. Low-frequency radio constraints on the synchrotron cosmic web. Mon. Not. R. Astron. Soc. 2017, 467, 4914-4936. [CrossRef]

41. Vacca, V.; Murgia, M.; Govoni, F.; Loi, F.; Vazza, F.; Finoguenov, A.; Carretti, E.; Feretti, L.; Giovannini, G.; Concu, R.; et al. Observations of a nearby filament of galaxy clusters with the Sardinia Radio Telescope. Mon. Not. R. Astron. Soc. 2018, 479, 776-806. [CrossRef]

42. Vernstrom, T.; Gaensler, B.M.; Rudnick, L.; Andernach, H. Differences in Faraday Rotation between Adjacent Extragalactic Radio Sources as a Probe of Cosmic Magnetic Fields. Astrophys. J. 2019, 878, 92. [CrossRef]

43. Govoni, F.; Orrù, E.; Bonafede, A.; Iacobelli, M.; Paladino, R.; Vazza, F.; Murgia, M.; Vacca, V.; Giovannini,-G.; Feretti, L.; et al. A radio ridge connecting two galaxy clusters in a filament of the cosmic web. Science 2019, 364, 981-984. [CrossRef] [PubMed]

44. Pshirkov, M.S.; Tinyakov, P.G.; Urban, F.R. New Limits on Extragalactic Magnetic Fields from Rotation Measures. Phys. Rev. Lett. 2016, 116, 191302. [CrossRef] [PubMed]

45. Ravi, V.; Shannon, R.M.; Bailes, M.; Bannister, K.; Bhandari, S.; Bhat, N.D.R.; Burke-Spolaor, S.; Caleb, M.; Flynn, C.; Jameson, A.; et al. The magnetic field and turbulence of the cosmic web measured using a brilliant fast radio burst. Science 2016, 354, 1249-1252. [CrossRef]

46. Hackstein, S.; Brüggen, M.; Vazza, F.; Gaensler, B.M.; Heesen, V. Fast radio burst dispersion measures and rotation measures and the origin of intergalactic magnetic fields. Mon. Not. R. Astron. Soc. 2019, 488, 4220-4238. [CrossRef]

47. O'Sullivan, S.P.; Machalski, J.; Van Eck, C.L.; Heald, G.; Brüggen, M.; Fynbo, J.P.U.; Heintz, K.E.; Lara-Lopez, M.A.; Vacca, V.; Hardcastle, M.J.; et al. The intergalactic magnetic field probed by a giant radio galaxy. Astron. Astrophys. 2019, 622, A16. [CrossRef]

48. O'Sullivan, S.P.; Brüggen, M.; Vazza, F.; Carretti, E.; Locatelli, N.; Stuardi, C.; Vacca, V.; Vernstrom, T.; Heald, G.; Horellou, C.; et al. New constraints on the magnetization of the cosmic web using LOFAR Faraday rotation observations. arXiv 2020, arXiv:astro-ph.CO/2002.06924.

49. Akahori, T.; Gaensler, B.M.; Ryu, D. Statistical Techniques for Detecting the Intergalactic Magnetic Field from Large Samples of Extragalactic Faraday Rotation Data. Astrophys. J. 2014, 790, 123. [CrossRef]

50. Akahori, T.; Ryu, D.; Gaensler, B.M. Fast Radio Bursts as Probes of Magnetic Fields in the Intergalactic Medium. Astrophys. J. 2016, 824, 105. [CrossRef]

51. Vazza, F.; Brüggen, M.; Gheller, C.; Hackstein, S.; Wittor, D.; Hinz, P.M. Simulations of extragalactic magnetic fields and of their observables. Class. Quantum Gravity 2017, 34, 234001. [CrossRef]

52. Locatelli, N.; Vazza, F.; Domínguez-Fernández, P. The Challenge of Detecting Intracluster Filaments with Faraday Rotation. Galaxies 2018, 6, 128. [CrossRef]

53. Vazza, F.; Brüggen, M.; Hinz, P.M.; Wittor, D.; Locatelli, N.; Gheller, C. Probing the origin of extragalactic magnetic fields with Fast Radio Bursts. Mon. Not. R. Astron. Soc. 2018, 480, 3907-3915. [CrossRef]

54. Macquart, J.P.; Keane, E.; Grainge, K.; McQuinn, M.; Fender, R.; Hessels, J.; Deller, A.; Bhat, R.; Breton, R.; Chatterjee, S.; et al. Fast Transients at Cosmological Distances with the SKA. In Advancing Astrophysics with the Square Kilometre Array (AASKA14); Sissa Medialab: Trieste, Italy, 2015; p. 55.

55. Subramanian, K. The origin, evolution and signatures of primordial magnetic fields. Rep. Prog. Phys. 2016, 79, 076901. [CrossRef] [PubMed] 
56. Schleicher, D.R.G.; Miniati, F. Primordial magnetic field constraints from the end of reionization. Mon. Not. R. Astron. Soc. 2011, 418, L143-L147. [CrossRef]

57. Widrow, L.M.; Ryu, D.; Schleicher, D.R.G.; Subramanian, K.; Tsagas, C.G.; Treumann, R.A. The First Magnetic Fields. Space Sci. Rev. 2012, 166, 37-70. [CrossRef]

58. Langer, M.; Durrive, J.B. Magnetizing the Cosmic Web during Reionization. Galaxies 2018, 6, 124. [CrossRef]

59. Koopmans, L.; Pritchard, J.; Mellema, G.; Aguirre, J.; Ahn, K.; Barkana, R.; van Bemmel, I.; Bernardi, G.; Bonaldi, A.; Briggs, F.; et al. The Cosmic Dawn and Epoch of Reionisation with SKA. In Advancing Astrophysics with the Square Kilometre Array (AASKA14); Sissa Medialab: Trieste, Italy, 2015; p. 1.

60. Minoda, T.; Hasegawa, K.; Tashiro, H.; Ichiki, K.; Sugiyama, N. Thermal Sunyaev-Zel'dovich Effect in the IGM due to Primordial Magnetic Fields. Galaxies 2018, 6, 143. [CrossRef]

61. van Weeren, R.J.; de Gasperin, F.; Akamatsu, H.; Brüggen, M.; Feretti, L.; Kang, H.; Stroe, A.; Zandanel, F. Diffuse Radio Emission from Galaxy Clusters. Space Sci. Rev. 2019, 215, 16. [CrossRef]

62. Govoni, F.; Murgia, M.; Xu, H.; Li, H.; Norman, M.L.; Feretti, L.; Giovannini, G.; Vacca, V. Polarization of cluster radio halos with upcoming radio interferometers. Astron. Astrophys. 2013, 554, A102. [CrossRef]

63. Kang, H.; Ryu, D. Diffusive Shock Acceleration at Cosmological Shock Waves. Astrophys. J. 2013, 764, 95. [CrossRef]

64. Brunetti, G.; Lazarian, A. Stochastic reacceleration of relativistic electrons by turbulent reconnection: a mechanism for cluster-scale radio emission? Mon. Not. R. Astron. Soc. 2016, 458, 2584-2595. [CrossRef]

65. Dolag, K. Properties of Simulated Magnetized Galaxy Clusters. Constructing the Universe with Clusters of Galaxies. arXiv 2000, arXiv:astro-ph/astro-ph/0010149.

66. Vazza, F.; Brunetti, G.; Brüggen, M.; Bonafede, A. Resolved magnetic dynamo action in the simulated intracluster medium. Mon. Not. R. Astron. Soc. 2018, 474, 1672-1687. [CrossRef]

67. Vacca, V.; Murgia, M.; Govoni, F.; Enßlin, T.; Oppermann, N.; Feretti, L.; Giovannini, G.; Loi, F. Magnetic Fields in Galaxy Clusters and in the Large-Scale Structure of the Universe. Galaxies 2018, 6, 142. [CrossRef]

68. Govoni, F.; Murgia, M.; Vacca, V.; Loi, F.; Girardi, M.; Gastaldello, F.; Giovannini, G.; Feretti, L.; Paladino, R.; Carretti, E.; et al. Sardinia Radio Telescope observations of Abell 194. The intra-cluster magnetic field power spectrum. Astron. Astrophys. 2017, 603, A122. [CrossRef]

69. Loi, F.; Murgia, M.; Govoni, F.; Vacca, V.; Bonafede, A.; Ferrari, C.; Prandoni, I.; Feretti, L.; Giovannini, G.; Li, H. Rotation measure synthesis applied to synthetic SKA images of galaxy clusters. Mon. Not. R. Astron. Soc. 2019, 490, 4841-4857. [CrossRef]

70. Markevitch, M.; Gonzalez, A.H.; Clowe, D.; Vikhlinin, A.; Forman, W.; Jones, C.; Murray, S.; Tucker, W. Direct Constraints on the Dark Matter Self-Interaction Cross Section from the Merging Galaxy Cluster 1E 0657-56. Astrophys. J. 2004, 606, 819-824. [CrossRef]

71. Spekkens, K.; Mason, B.S.; Aguirre, J.E.; Nhan, B. A Deep Search for Extended Radio Continuum Emission from Dwarf Spheroidal Galaxies: Implications for Particle Dark Matter. Astrophys. J. 2013, 773, 61. [CrossRef]

72. Regis, M.; Colafrancesco, S.; Profumo, S.; de Blok, W.; Massardi, M.; Richter, L. Local Group dSph radio survey with ATCA (III): Constraints on Particle Dark Matter. J. Cosmol. Astropart. Phys. 2014, 10, 016. [CrossRef]

73. Regis, M.; Richter, L.; Colafrancesco, S. Dark matter in the Reticulum II dSph: a radio search. J. Cosmol. Astropart. Phys. 2017, 2017, 025. [CrossRef]

74. Vollmann, M.; Heesen, V.; Shimwell, T.; Hardcastle, M.J.; Brüggen, M.; Sigl, G.; Röttgering, H. Radio constraints on dark matter annihilation in Canes Venatici I with LOFAR. arXiv 2019, arXiv:1909.12355.

75. Cook, R.H.W.; Seymour, N.; Spekkens, K.; Hurley-Walker, N.; Hancock, P.J.; Bell, M.E.; Callingham, J.R.; For, B.Q.; Franzen, T.M.O.; Gaensler, B.M.; et al. Searching for dark matter signals from local dwarf spheroidal galaxies at low radio frequencies in the GLEAM survey. Mon. Not. R. Astron. Soc. 2020, 494, 135-145. [CrossRef]

76. Lorimer, D.R. A decade of fast radio bursts. Nat. Astron. 2018, 2, 860-864. [CrossRef]

77. Lorimer, D.R.; Bailes, M.; McLaughlin, M.A.; Narkevic, D.J.; Crawford, F. A Bright Millisecond Radio Burst of Extragalactic Origin. Science 2007, 318, 777. [CrossRef]

78. Chatterjee, S.; Law, C.J.; Wharton, R.S.; Burke-Spolaor, S.; Hessels, J.W.T.; Bower, G.C.; Cordes, J.M.; Tendulkar, S.P.; Bassa, C.G.; Demorest, P.; et al. A direct localization of a fast radio burst and its host. Nature 2017, 541, 58-61. [CrossRef] [PubMed] 
79. Bannister, K.W.; Deller, A.T.; Phillips, C.; Macquart, J.P.; Prochaska, J.X.; Tejos, N.; Ryder, S.D.; Sadler, E.M.; Shannon, R.M.; Simha, S.; et al. A single fast radio burst localized to a massive galaxy at cosmological distance. Science 2019, 365, 565-570. [CrossRef]

80. Michilli, D.; Seymour, A.; Hessels, J.W.T.; Spitler, L.G.; Gajjar, V.; Archibald, A.M.; Bower, G.C.; Chatterjee, S.; Cordes, J.M.; Gourdji, K.; et al. An extreme magneto-ionic environment associated with the fast radio burst source FRB 121102. Nature 2018, 553, 182-185. [CrossRef]

81. Wilkinson, P. The SKA and the Unknown Unknowns. In Advancing Astrophysics with the Square Kilometre Array (AASKA14); Sissa Medialab: Trieste, Italy, 2015; p. 65.

82. Krumholz, M.R.; Federrath, C. The Role of Magnetic Fields in Setting the Star Formation Rate and the Initial Mass Function. Front. Astron. Space Sci. 2019, 6, 7. [CrossRef]

83. Beck, R. Magnetic fields in spiral galaxies. Astron. Astrophys. Rev. 2015, 24, 4. [CrossRef]

84. Arshakian, T.G.; Beck, R.; Krause, M.; Sokoloff, D. Evolution of magnetic fields in galaxies and future observational tests with the Square Kilometre Array. Astron. Astrophys. 2009, 494, 21-32. [CrossRef]

85. Beck, A.M.; Lesch, H.; Dolag, K.; Kotarba, H.; Geng, A.; Stasyszyn, F.A. Origin of strong magnetic fields in Milky Way-like galactic haloes. Mon. Not. R. Astron. Soc. 2012, 422, 2152-2163. [CrossRef]

86. Martin-Alvarez, S.; Devriendt, J.; Slyz, A.; Teyssier, R. A three-phase amplification of the cosmic magnetic field in galaxies. Mon. Not. R. Astron. Soc. 2018, 479, 3343-3365. [CrossRef]

87. Rodrigues, L.F.S.; Chamandy, L.; Shukurov, A.; Baugh, C.M.; Taylor, A.R. Evolution of galactic magnetic fields. Mon. Not. R. Astron. Soc. 2019, 483, 2424-2440. [CrossRef]

88. Bernet, M.L.; Miniati, F.; Lilly, S.J.; Kronberg, P.P.; Dessauges-Zavadsky, M. Strong magnetic fields in normal galaxies at high redshift. Nature 2008, 454, 302-304. [CrossRef] [PubMed]

89. Farnes, J.S.; O'Sullivan, S.P.; Corrigan, M.E.; Gaensler, B.M. Faraday Rotation from Magnesium II Absorbers toward Polarized Background Radio Sources. Astrophys. J. 2014, 795, 63. [CrossRef]

90. Mao, S.A.; Carilli, C.; Gaensler, B.M.; Wucknitz, O.; Keeton, C.; Basu, A.; Beck, R.; Kronberg, P.P.; Zweibel, E. Detection of microgauss coherent magnetic fields in a galaxy five billion years ago. Nat. Astron. 2017, 1, 621-626. [CrossRef]

91. Sur, S.; Bhat, P.; Subramanian, K. Faraday rotation signatures of fluctuation dynamos in young galaxies. Mon. Not. R. Astron. Soc. 2018, 475, L72-L76. [CrossRef]

92. Beck, R.; Chamandy, L.; Elson, E.; Blackman, E.G. Synthesizing Observations and Theory to Understand Galactic Magnetic Fields: Progress and Challenges. Galaxies 2019, 8, 4. [CrossRef]

93. Heald, G.; Braun, R.; Edmonds, R. The Westerbork SINGS survey. II Polarization, Faraday rotation, and magnetic fields. Astron. Astrophys. 2009, 503, 409-435. [CrossRef]

94. Irwin, J.; Beck, R.; Benjamin, R.A.; Dettmar, R.J.; English, J.; Heald, G.; Henriksen, R.N.; Johnson, M.; Krause, M.; Li, J.T.; et al. Continuum Halos in Nearby Galaxies: An EVLA Survey (CHANG-ES). I. Introduction to the Survey. Astrophys. J. 2012, 144, 43. [CrossRef]

95. Mulcahy, D.D.; Beck, R.; Heald, G.H. Resolved magnetic structures in the disk-halo interface of NGC 628. Astron. Astrophys. 2017, 600, A6. [CrossRef]

96. Mulcahy, D.D.; Horneffer, A.; Beck, R.; Krause, M.; Schmidt, P.; Basu, A.; Chyży, K.T.; Dettmar, R.J.; Haverkorn, M.; Heald, G.; et al. Investigation of the cosmic ray population and magnetic field strength in the halo of NGC 891. Astron. Astrophys. 2018, 615, A98. [CrossRef]

97. Irwin, J.; Damas-Segovia, A.; Krause, M.; Miskolczi, A.; Li, J.; Stein, Y.; English, J.; Henriksen, R.; Beck, R.; Wiegert, T.; et al. CHANG-ES: XVIII-The CHANG-ES Survey and Selected Results. Galaxies 2019, 7, 42. [CrossRef]

98. Heesen, V.; Buie, E.I.; Huff, C.J.; Perez, L.A.; Woolsey, J.G.; Rafferty, D.A.; Basu, A.; Beck, R.; Brinks, E.; Horellou, C.; et al. Calibrating the relation of low-frequency radio continuum to star formation rate at $1 \mathrm{kpc}$ scale with LOFAR. Astron. Astrophys. 2019, 622, A8. [CrossRef]

99. Krause, M.; Irwin, J.; Wiegert, T.; Miskolczi, A.; Damas-Segovia, A.; Beck, R.; Li, J.T.; Heald, G.; Müller, P.; Stein, Y.; Rand, R.J.; Heesen, V.; Walterbos, R.A.M.; Dettmar, R.J.; Vargas, C.J.; English, J.; Murphy, E.J. CHANG-ES. IX. Radio scale heights and scale lengths of a consistent sample of 13 spiral galaxies seen edge-on and their correlations. Astron. Astrophys. 2018, 611, A72. [CrossRef]

100. Heesen, V.; Krause, M.; Beck, R.; Adebahr, B.; Bomans, D.J.; Carretti, E.; Dumke, M.; Heald, G.; Irwin, J.; Koribalski, B.S.; et al. Radio haloes in nearby galaxies modelled with 1D cosmic ray transport using SPINNAKER. Mon. Not. R. Astron. Soc. 2018, 476, 158-183. [CrossRef] 
101. Bertone, S.; Vogt, C.; Enßlin, T. Magnetic field seeding by galactic winds. Mon. Not. R. Astron. Soc. 2006, 370, 319-330. [CrossRef]

102. Pakmor, R.; van de Voort, F.; Bieri, R.; Gomez, F.A.; Grand, R.J.J.; Guillet, T.; Marinacci, F.; Pfrommer, C.; Simpson, C.M.; Springel, V. Magnetising the circumgalactic medium of disk galaxies. arXiv 2019, arXiv:1911.11163.

103. Berlok, T.; Pfrommer, C. The impact of magnetic fields on cold streams feeding galaxies. Mon. Not. R. Astron. Soc. 2019, 489, 3368-3384. [CrossRef]

104. Vogelsberger, M.; Marinacci, F.; Torrey, P.; Puchwein, E. Cosmological simulations of galaxy formation. Nat. Rev. Phys. 2020, 2, 42-66. [CrossRef]

105. Mulcahy, D.D.; Horneffer, A.; Beck, R.; Heald, G.; Fletcher, A.; Scaife, A.; Adebahr, B.; Anderson, J.M.; Bonafede, A.; Brüggen, M.; et al. The nature of the low-frequency emission of M 51. First observations of a nearby galaxy with LOFAR. Astron. Astrophys. 2014, 568, A74. [CrossRef]

106. Neld, A.; Horellou, C.; Mulcahy, D.D.; Beck, R.; Bourke, S.; Carozzi, T.D.; Chyży, K.T.; Conway, J.E.; Farnes, J.S.; Fletcher, A.; et al. Reliable detection and characterization of low-frequency polarized sources in the LOFAR M51 field. Astron. Astrophys. 2018, 617, A136. [CrossRef]

107. Nikiel-Wroczyński, B.; Berger, A.; Herrera Ruiz, N.; Bomans, D.J.; Blex, S.; Horellou, C.; Paladino, R.; Becker, A.; Miskolczi, A.; Beck, R.; et al. Exploring the properties of low-frequency radio emission and magnetic fields in a sample of compact galaxy groups using the LOFAR Two-Metre Sky Survey (LoTSS). Astron. Astrophys. 2019, 622, A23. [CrossRef]

108. Kaczmarek, J.F.; Purcell, C.R.; Gaensler, B.M.; McClure-Griffiths, N.M.; Stevens, J. Detection of a Coherent Magnetic Field in the Magellanic Bridge through Faraday Rotation. Mon. Not. R. Astron. Soc. 2017, 467, 1776-1794. [CrossRef]

109. Basu, A.; Mao, S.A.; Kepley, A.A.; Robishaw, T.; Zweibel, E.G.; Gallagher, J.S., III. Detection of an 20 kpc coherent magnetic field in the outskirt of merging spirals: the Antennae galaxies. Mon. Not. R. Astron. Soc. 2017, 464, 1003-1017. [CrossRef]

110. Ulrich, M.H.; Maraschi, L.; Urry, C.M. Variability of Active Galactic Nuclei. Annu. Rev. 1997, 35, 445-502. [CrossRef]

111. Balbus, S.A.; Hawley, J.F. A Powerful Local Shear Instability in Weakly Magnetized Disks. I. Linear Analysis. Astrophys. J. 1991, 376, 214. [CrossRef]

112. Event Horizon Telescope Collaboration; Akiyama, K.; Alberdi, A.; Alef, W.; Asada, K.; Azulay, R.; Baczko, A.K.; Ball, D.; Baloković, M.; Barrett, J.; et al. First M87 Event Horizon Telescope Results. I. The Shadow of the Supermassive Black Hole. Astrophys. J. 2019, 875, L1. [CrossRef]

113. Event Horizon Telescope Collaboration; Akiyama, K.; Alberdi, A.; Alef, W.; Asada, K.; Azulay, R.; Baczko, A.K.; Ball, D.; Baloković, M.; Barrett, J.; et al. First M87 Event Horizon Telescope Results. VI. The Shadow and Mass of the Central Black Hole. Astrophys. J. 2019, 875, L6. [CrossRef]

114. Gabuzda, D.C.; Nagle, M.; Roche, N. The jets of AGN as giant coaxial cables. Astron. Astrophys. 2018, 612, A67. [CrossRef]

115. Furlanetto, S.R.; Loeb, A. Intergalactic Magnetic Fields from Quasar Outflows. Astrophys. J. 2001, 556, 619-634. [CrossRef]

116. Calzadilla, M.S.; McDonald, M.; Bayliss, M.; Benson, B.A.; Bleem, L.E.; Brodwin, M.; Edge, A.C.; Floyd, B.; Gupta, N.; Hlavacek-Larrondo, J.; et al. Discovery of a Powerful $<10^{61}$ erg AGN Outburst in the Distant Galaxy Cluster SPT-CLJ0528-5300. Astrophys. J. 2019, 887, L17. [CrossRef]

117. Giacintucci, S.; Markevitch, M.; Johnston-Hollitt, M.; Wik, D.R.; Wang, Q.H.S.; Clarke, T.E. Discovery of a Giant Radio Fossil in the Ophiuchus Galaxy Cluster. Astrophys. J. 2020, 891, 1. [CrossRef]

118. Roy, S.; Pramesh Rao, A.; Subrahmanyan, R. Magnetic field near the central region of the Galaxy: rotation measure of extragalactic sources. Astron. Astrophys. 2008, 478, 435-442. [CrossRef]

119. Xu, J.; Han, J.L. Magnetic fields in the solar vicinity and in the Galactic halo. Mon. Not. R. Astron. Soc. 2019, 486, 4275-4289. [CrossRef]

120. Sobey, C.; Bilous, A.V.; Grießmeier, J.M.; Hessels, J.W.T.; Karastergiou, A.; Keane, E.F.; Kondratiev, V.I.; Kramer, M.; Michilli, D.; Noutsos, A.; et al. Low-frequency Faraday rotation measures towards pulsars using LOFAR: probing the 3D Galactic halo magnetic field. Mon. Not. R. Astron. Soc. 2019, 484, 3646-3664. [CrossRef] 
121. Rodrigues, L.F.S.; Sarson, G.R.; Shukurov, A.; Bushby, P.J.; Fletcher, A. The Parker Instability in Disk Galaxies. Astrophys. J. 2016, 816, 2. [CrossRef]

122. Pakmor, R.; Springel, V. Simulations of magnetic fields in isolated disc galaxies. Mon. Not. R. Astron. Soc. 2013, 432, 176-193. [CrossRef]

123. Chamandy, L.; Shukurov, A.; Taylor, A.R. New Insights on Galactic Dynamos. arXiv 2018, arXiv:1810.07246.

124. Brandenburg, A.; Brüggen, M. Hemispheric handedness in the Galactic synchrotron polarization foreground. arXiv 2020, arXiv:2003.14178.

125. Gaensler, B.M.; Haverkorn, M.; Burkhart, B.; Newton-McGee, K.J.; Ekers, R.D.; Lazarian, A.; McClure-Griffiths, N.M.; Robishaw, T.; Dickey, J.M.; Green, A.J. Low-Mach-number turbulence in interstellar gas revealed by radio polarization gradients. Nature 2011, 478, 214-217. [CrossRef] [PubMed]

126. Basu, A.; Fletcher, A.; Mao, S.A.; Burkhart, B.; Beck, R.; Schnitzeler, D. An In-depth Investigation of Faraday Depth Spectrum Using Synthetic Observations of Turbulent MHD Simulations. Galaxies 2019, 7, 89. [CrossRef]

127. Costa, A.H.; Spangler, S.R.; Sink, J.R.; Brown, S.; Mao, S.A. Denser Sampling of the Rosette Nebula with Faraday Rotation Measurements: Improved Estimates of Magnetic Fields in H II Regions. Astrophys. J. 2016, 821, 92. [CrossRef]

128. West, J.L.; Safi-Harb, S.; Ferrand, G. The connection between supernova remnants and the Galactic magnetic field: An analysis of quasi-parallel and quasi-perpendicular cosmic-ray acceleration for the axisymmetric sample. Astron. Astrophys. 2017, 597, A121. [CrossRef]

129. Costa, A.H.; Spangler, S.R. A Faraday Rotation Study of the Stellar Bubble and H II Region Associated with the W4 Complex. Astrophys. J. 2018, 865, 65. [CrossRef]

130. Abbate, F.; Possenti, A.; Tiburzi, C.; Barr, E.; van Straten, W.; Ridolfi, A.; Freire, P. Constraints on the magnetic field in the Galactic halo from globular cluster pulsars. Nat. Astron. 2020. [CrossRef]

131. Betti, S.K.; Hill, A.S.; Mao, S.A.; Gaensler, B.M.; Lockman, F.J.; McClure-Griffiths, N.M.; Benjamin, R.A. Constraining the Magnetic Field of the Smith High-velocity Cloud Using Faraday Rotation. Astrophys. J. 2019, 871, 215. [CrossRef]

132. Wolleben, M.; Landecker, T.L.; Carretti, E.; Dickey, J.M.; Fletcher, A.; McClure-Griffiths, N.M.; McConnell, D.; Thomson, A.J.M.; Hill, A.S.; Gaensler, B.M.; et al. The Global Magneto-Ionic Medium Survey: Polarimetry of the Southern Sky from 300 to $480 \mathrm{MHz}$. Astrophys. J. 2019, 158, 44. [CrossRef]

133. Carretti, E.; Haverkorn, M.; Staveley-Smith, L.; Bernardi, G.; Gaensler, B.M.; Kesteven, M.J.; Poppi, S.; Brown, S.; Crocker, R.M.; Purcell, C.; et al. S-band Polarization All-Sky Survey (S-PASS): survey description and maps. Mon. Not. R. Astron. Soc. 2019, 489, 2330-2354. [CrossRef]

134. Dickey, J.M.; Landecker, T.L.; Thomson, A.J.M.; Wolleben, M.; Sun, X.; Carretti, E.; Douglas, K.; Fletcher, A.; Gaensler, B.M.; Gray, A.; et al. The Galactic Magneto-ionic Medium Survey: Moments of the Faraday Spectra. Astrophys. J. 2019, 871, 106. [CrossRef]

135. Thomson, A.J.M.; Landecker, T.L.; Dickey, J.M.; McClure-Griffiths, N.M.; Wolleben, M.; Carretti, E.; Fletcher, A.; Federrath, C.; Hill, A.S.; Mao, S.A.; et al. Through thick or thin: multiple components of the magneto-ionic medium towards the nearby H II region Sharpless 2-27 revealed by Faraday tomography. Mon. Not. R. Astron. Soc. 2019, 487, 4751-4767. [CrossRef]

136. Farrar, G.R.; Jansson, R.; Feain, I.J.; Gaensler, B.M. Galactic magnetic deflections and Centaurus A as a UHECR source. J. Cosmol. Astropart. Phys. 2013, 2013, 023. [CrossRef]

137. Boulanger, F.; Enßlin, T.; Fletcher, A.; Girichides, P.; Hackstein, S.; Haverkorn, M.; Hörandel, J.R.; Jaffe, T.; Jasche, J.; Kachelrieß, M.; et al. IMAGINE: a comprehensive view of the interstellar medium, Galactic magnetic fields and cosmic rays. J. Cosmol. Astropart. Phys. 2018, 2018, 049. [CrossRef]

138. Poidevin, F.; Rubino-Martin, J.A.; Genova-Santos, R.; Rebolo, R.; Aguiar, M.; Gomez-Renasco, F.; Guidi, F.; Gutierrez, C.; Hoyland, R.J.; Lopez-Caraballo, C.; et al. The QUIJOTE Experiment: Prospects for CMB B-MODE polarization detection and foregrounds characterization. arXiv 2018, arXiv:1802.04594.

139. Krachmalnicoff, N.; Carretti, E.; Baccigalupi, C.; Bernardi, G.; Brown, S.; Gaensler, B.M.; Haverkorn, M.; Kesteven, M.; Perrotta, F.; Poppi, S.; et al. S-PASS view of polarized Galactic synchrotron at $2.3 \mathrm{GHz}$ as a contaminant to CMB observations. Astron. Astrophys. 2018, 618, A166. [CrossRef]

140. Basu, A.; Schwarz, D.J.; Klöckner, H.R.; von Hausegger, S.; Kramer, M.; Wieching, G.; Burkhart, B. CMB foreground measurements through broad-band radio spectro-polarimetry: prospects of the SKA-MPG telescope. Mon. Not. R. Astron. Soc. 2019, 488, 1618-1634. [CrossRef] 
141. de la Hoz, E.; Vielva, P.; Barreiro, R.B.; Martínez-González, E. On the Detection of CMB B-modes from Ground at Low Frequency. arXiv 2020, arXiv:2002.12206.

142. Hutschenreuter, S.; Enßlin, T.A. The Galactic Faraday depth sky revisited. Astron. Astrophys. 2020, 633, A150. [CrossRef]

143. Sun, X.H.; Landecker, T.L.; Gaensler, B.M.; Carretti, E.; Reich, W.; Leahy, J.P.; McClure-Griffiths, N.M.; Crocker, R.M.; Wolleben, M.; Haverkorn, M.; et al. Faraday Tomography of the North Polar Spur: Constraints on the Distance to the Spur and on the Magnetic Field of the Galaxy. Astrophys. J. 2015, 811, 40. [CrossRef]

144. Crutcher, R.M. Magnetic Fields in Molecular Clouds. Annu. Rev. 2012, 50, 29-63. [CrossRef]

145. Li, H.B.; Goodman, A.; Sridharan, T.K.; Houde, M.; Li, Z.Y.; Novak, G.; Tang, K.S. The Link Between Magnetic Fields and Cloud/Star Formation. In Protostars and Planets VI; Beuther, H., Klessen, R.S., Dullemond, C.P., Henning, T., Eds.; University of Arizona Press: University of Arizona Press: Tucson, AZ, USA, 2014; p. 101. [CrossRef]

146. Hennebelle, P.; Inutsuka, S.i. The role of magnetic field in molecular cloud formation and evolution. Front. Astron. Space Sci. 2019, 6, 5. [CrossRef]

147. Federrath, C.; Klessen, R.S. The Star Formation Rate of Turbulent Magnetized Clouds: Comparing Theory, Simulations, and Observations. Astrophys. J. 2012, 761, 156. [CrossRef]

148. Federrath, C. Inefficient star formation through turbulence, magnetic fields and feedback. Mon. Not. R. Astron. Soc. 2015, 450, 4035-4042. [CrossRef]

149. Pudritz, R.E.; Ray, T.P. The Role of Magnetic Fields in Protostellar Outflows and Star Formation. Front. Astron. Space Sci. 2019, 6, 54. [CrossRef]

150. Wurster, J.; Li, Z.Y. The role of magnetic fields in the formation of protostellar discs. Front. Astron. Space Sci. 2018, 5, 39. [CrossRef]

151. Soler, J.D. Using Herschel and Planck observations to delineate the role of magnetic fields in molecular cloud structure. Astron. Astrophys. 2019, 629, A96. [CrossRef]

152. Brandenburg, A.; Subramanian, K. Astrophysical magnetic fields and nonlinear dynamo theory. Phys. Rep. 2005, 417, 1-209. [CrossRef]

153. Houde, M.; Rao, R.; Vaillancourt, J.E.; Hildebrand, R.H. Dispersion of Magnetic Fields in Molecular Clouds. III. Astrophys. J. 2011, 733, 109. [CrossRef]

154. Burkhart, B.; Lazarian, A.; Gaensler, B.M. Properties of Interstellar Turbulence from Gradients of Linear Polarization Maps. Astrophys. J. 2012, 749, 145. [CrossRef]

155. Falgarone, E.; Momferratos, G.; Lesaffre, P., The Intermittency of ISM Turbulence: What Do the Observations Tell Us? In Magnetic Fields in Diffuse Media; Lazarian, A., de Gouveia Dal Pino, E.M., Melioli, C., Eds.; Astrophysics and Space Science Library, Springer: Basel, Switzerland, 2015; Volume 407. [CrossRef]

156. Federrath, C. The role of turbulence, magnetic fields and feedback for star formation. J. Phys. Conf. Ser. 2016, 719, 12002. [CrossRef]

157. Padovani, M.; Galli, D. Synchrotron emission in molecular cloud cores: the SKA view. Astron. Astrophys. 2018, 620, L4. [CrossRef]

158. André, P.; Di Francesco, J.; Ward-Thompson, D.; Inutsuka, S.I.; Pudritz, R.E.; Pineda, J.E. From Filamentary Networks to Dense Cores in Molecular Clouds: Toward a New Paradigm for Star Formation. In Protostars and Planets VI; Beuther, H., Klessen, R.S., Dullemond, C.P., Henning, T., Eds.; University of Arizona Press: Tucson, AZ, USA, 2014; p. 27. [CrossRef]

159. Poidevin, F. Analysis of Galactic molecular cloud polarization maps: a review of the methods. Eur. Phys. J. Web Conf. 2020, 228, 19. [CrossRef]

160. Green, J.A.; McClure-Griffiths, N.M.; Caswell, J.L.; Robishaw, T.; Harvey-Smith, L. MAGMO: coherent magnetic fields in the star-forming regions of the Carina-Sagittarius spiral arm tangent. Mon. Not. R. Astron. Soc. 2012, 425, 2530-2547. [CrossRef]

161. Ogbodo, C.S.; Green, J.A.; Dawson, J.R.; Breen, S.L.; Mao, S.A.; McClure-Griffiths, N.M.; Robishaw, T.; Harvey-Smith, L. MAGMO: polarimetry of 1720-MHz OH masers towards southern star-forming regions. Mon. Not. R. Astron. Soc. 2020, 493, 199-233. [CrossRef]

162. Tahani, M.; Plume, R.; Brown, J.C.; Kainulainen, J. Helical magnetic fields in molecular clouds?. A new method to determine the line-of-sight magnetic field structure in molecular clouds. Astron. Astrophys. 2018, 614, A100. [CrossRef] 
163. Tahani, M.; Plume, R.; Brown, J.C.; Soler, J.D.; Kainulainen, J. Could bow-shaped magnetic morphologies surround filamentary molecular clouds?. The 3D magnetic field structure of Orion-A. Astron. Astrophys. 2019, 632, A68. [CrossRef]

164. Shang, H.; Allen, A.; Li, Z.Y.; Liu, C.F.; Chou, M.Y.; Anderson, J. A Unified Model for Bipolar Outflows from Young Stars. Astrophys. J. 2006, 649, 845-855. [CrossRef]

165. Gerrard, I.A.; Federrath, C.; Kuruwita, R. The role of initial magnetic field structure in the launching of protostellar jets. Mon. Not. R. Astron. Soc. 2019, 485, 5532-5542. [CrossRef]

166. Hu, X.; Zhu, Z.; Okuzumi, S.; Bai, X.N.; Wang, L.; Tomida, K.; Stone, J.M. Nonideal MHD Simulation of HL Tau Disk: Formation of Rings. Astrophys. J. 2019, 885, 36. [CrossRef]

167. Mori, S.; Bai, X.N.; Okuzumi, S. Temperature Structure in the Inner Regions of Protoplanetary Disks: Inefficient Accretion Heating Controlled by Nonideal Magnetohydrodynamics. Astrophys. J. 2019, 872, 98. [CrossRef]

168. Hull, C.L.H.; Carrasco-González, C.; Williams, P.K.G.; Girart, J.M.; Robishaw, T.; Galván-Madrid, R.; Bourke, T. Magnetic Fields in Forming Stars with the ngVLA. In Science with a Next Generation Very Large Array; Astronomical Society of the Pacific Conference Series; Murphy, E., Ed.; Astronomical Society of the Pacific: San Francisco, CA, USA, 2018; Volume 517, p. 357.

169. Guillet, V.; Girart, J.M.; Maury, A.J.; Alves, F.O. Polarized emission by aligned grains in the Mie regime: Application to protoplanetary disks observed by ALMA. Astron. Astrophys. 2020, 634, L15. [CrossRef]

170. Stil, J.; Wityk, N.; Ouyed, R.; Taylor, A.R. Three-dimensional Simulations of Magnetized Superbubbles: New Insights into the Importance of MHD Effects on Observed Quantities. Astrophys. J. 2009, 701, 330-347. [CrossRef]

171. Padovani, M.; Marcowith, A.; Sánchez-Monge, Á.; Meng, F.; Schilke, P. Non-thermal emission from cosmic rays accelerated in H II regions. Astron. Astrophys. 2019, 630, A72. [CrossRef]

172. Meng, F.; Sánchez-Monge, Á.; Schilke, P.; Padovani, M.; Marcowith, A.; Ginsburg, A.; Schmiedeke, A.; Schwörer, A.; DePree, C.; Veena, V.S.; et al. The physical and chemical structure of Sagittarius B2. V. Non-thermal emission in the envelope of Sgr B2. Astron. Astrophys. 2019, 630, A73. [CrossRef]

173. Kothes, R.; Brown, J.A. Probing interstellar magnetic fields with Supernova remnants. In Cosmic Magnetic Fields: From Planets, to Stars and Galaxies; Strassmeier, K.G., Kosovichev, A.G., Beckman, J.E., Eds.; IAU Symposium, Cambridge University Press: Cambridge, UK, 2009; Volume 259, pp. 75-80. [CrossRef]

174. West, J.L.; Safi-Harb, S.; Jaffe, T.; Kothes, R.; Landecker, T.L.; Foster, T. The connection between supernova remnants and the Galactic magnetic field: A global radio study of the axisymmetric sample. Astron. Astrophys. 2016, 587, A148. [CrossRef]

175. Howard, T.A.; Stovall, K.; Dowell, J.; Taylor, G.B.; White, S.M. Measuring the Magnetic Field of Coronal Mass Ejections Near the Sun Using Pulsars. Astrophys. J. 2016, 831, 208. [CrossRef]

176. Kooi, J.E.; Fischer, P.D.; Buffo, J.J.; Spangler, S.R. VLA Measurements of Faraday Rotation through Coronal Mass Ejections. Sol. Phys. 2017, 292, 56. [CrossRef]

177. Hurley-Walker, N.; Hancock, P.J. De-distorting ionospheric effects in the image plane. Astron. Comput. 2018, 25, 94-102. [CrossRef]

178. Sotomayor-Beltran, C.; Sobey, C.; Hessels, J.W.T.; de Bruyn, G.; Noutsos, A.; Alexov, A.; Anderson, J.; Asgekar, A.; Avruch, I.M.; Beck, R.; et al. Calibrating high-precision Faraday rotation measurements for LOFAR and the next generation of low-frequency radio telescopes. Astron. Astrophys. 2013, 552, A58. [CrossRef]

179. Porayko, N.K.; Noutsos, A.; Tiburzi, C.; Verbiest, J.P.W.; Horneffer, A.; Künsemöller, J.; Osłowski, S.; Kramer, M.; Schnitzeler, D.H.F.M.; Anderson, J.M.; et al. Testing the accuracy of the ionospheric Faraday rotation corrections through LOFAR observations of bright northern pulsars. Mon. Not. R. Astron. Soc. 2019, 483, 4100-4113. [CrossRef]

180. Oosterloo, T.; Verheijen, M.; van Cappellen, W. The latest on Apertif. In Proceedings of the ISKAF2010 Science Meeting, Assen, The Netherlands, 10-14 June 2010; p. 43,

181. Johnston, S.; Taylor, R.; Bailes, M.; Bartel, N.; Baugh, C.; Bietenholz, M.; Blake, C.; Braun, R.; Brown, J.; Chatterjee, S.; et al. Science with ASKAP. The Australian square-kilometre-array pathfinder. Exp. Astron. 2008, 22, 151-273. [CrossRef] 
182. Anderson, C.; Heald, G.; O'Sullivan, S.; Bunton, J.; Carretti, E.; Chippendale, A.; Collier, J.; Farnes, J.; Gaensler, B.; Harvey-Smith, L.; et al. The Extraordinary Linear Polarisation Structure of the Southern Centaurus A Lobe Revealed by ASKAP. Galaxies 2018, 6, 127. [CrossRef]

183. Jonas, J.; MeerKAT Team. The MeerKAT Radio Telescope. MeerKAT Science: On the Pathway to the SKA; Sissa Medialab: Trieste, Italy, 2016; p. 1.

184. Camilo, F. African star joins the radio astronomy firmament. Nat. Astron. 2018, 2, 594-594. [CrossRef]

185. Lacy, M.; Baum, S.A.; Chandler, C.J.; Chatterjee, S.; Clarke, T.E.; Deustua, S.; English, J.; Farnes, J.; Gaensler, B.M.; Gugliucci, N.; et al. The Karl G. Jansky Very Large Array Sky Survey (VLASS). Science Case and Survey Design. Publ. Astron. Soc. Pac. 2020, 132, 035001. [CrossRef]

186. Hobbs, G.; Manchester, R.N.; Dunning, A.; Jameson, A.; Roberts, P.; George, D.; Green, J.A.; Tuthill, J.; Toomey, L.; Kaczmarek, J.F.; et al. An ultra-wide bandwidth (704 to $4032 \mathrm{MHz}$ ) receiver for the Parkes radio telescope. Publ. Astron. Soc. Aust. 2020, 37, e012. [CrossRef]

187. Shanahan, R.; Lemmer, S.J.; Stil, J.M.; Beuther, H.; Wang, Y.; Soler, J.; Anderson, L.D.; Bigiel, F.; Glover, S.C.O.; Goldsmith, P.; et al. Strong Excess Faraday Rotation on the Inside of the Sagittarius Spiral Arm. Astrophys. J. 2019, 887, L7. [CrossRef]

188. Zhang, L.; Hobbs, G.; Manchester, R.N.; Li, D.; Wang, P.; Dai, S.; Wang, J.; Kaczmarek, J.F.; Cameron, A.D.; Toomey, L.; et al. Wide Bandwidth Observations of Pulsars C, D, and J in 47 Tucanae. Astrophys. J. 2019, 885, L37. [CrossRef]

189. Dai, S.; Johnston, S.; Kerr, M.; Camilo, F.O.; Cameron, A.; Toomey, L.; Kumamoto, H. Discovery of Millisecond Pulsars in the Globular Cluster Omega Centauri. Astrophys. J. 2020, 888, L18. [CrossRef]

190. van Haarlem, M.P.; Wise, M.W.; Gunst, A.W.; Heald, G.; McKean, J.P.; Hessels, J.W.T.; de Bruyn, A.G.; Nijboer, R.; Swinbank, J.; Fallows, R.; et al. LOFAR: The LOw-Frequency ARray. Astron. Astrophys. 2013, 556, A2. [CrossRef]

191. Tingay, S.J.; Goeke, R.; Bowman, J.D.; Emrich, D.; Ord, S.M.; Mitchell, D.A.; Morales, M.F.; Booler, T.; Crosse, B.; Wayth, R.B.; et al. The Murchison Widefield Array: The Square Kilometre Array Precursor at Low Radio Frequencies. Publ. Astron. Soc. Aust. 2013, 30, e007. [CrossRef]

192. Wayth, R.B.; Tingay, S.J.; Trott, C.M.; Emrich, D.; Johnston-Hollitt, M.; McKinley, B.; Gaensler, B.M.; Beardsley, A.P.; Booler, T.; Crosse, B.; et al. The Phase II Murchison Widefield Array: Design overview. Publ. Astron. Soc. Aust. 2018, 35, 33. [CrossRef]

193. Lenc, E.; Gaensler, B.M.; Sun, X.H.; Sadler, E.M.; Willis, A.G.; Barry, N.; Beardsley, A.P.; Bell, M.E.; Bernardi, G.; Bowman, J.D.; et al. Low-frequency Observations of Linearly Polarized Structures in the Interstellar Medium near the South Galactic Pole. Astrophys. J. 2016, 830, 38. [CrossRef]

194. Lenc, E.; Anderson, C.S.; Barry, N.; Bowman, J.D.; Cairns, I.H.; Farnes, J.S.; Gaensler, B.M.; Heald, G.; Johnston-Hollitt, M.; Kaplan, D.L.; et al. The Challenges of Low-Frequency Radio Polarimetry: Lessons from the Murchison Widefield Array. Publ. Astron. Soc. Aust. 2017, 34, e040. [CrossRef]

195. Riseley, C.J.; Lenc, E.; Van Eck, C.L.; Heald, G.; Gaensler, B.M.; Anderson, C.S.; Hancock, P.J.; Hurley-Walker, N.; Sridhar, S.S.; White, S.V. The POlarised GLEAM Survey (POGS) I: First results from a low-frequency radio linear polarisation survey of the southern sky. Publ. Astron. Soc. Aust. 2018, $35,43$. [CrossRef]

196. Van Eck, C.L.; Haverkorn, M.; Alves, M.I.R.; Beck, R.; Best, P.; Carretti, E.; Chyży, K.T.; Farnes, J.S.; Ferrière, K.; Hardcastle, M.J.; et al. Polarized point sources in the LOFAR Two-meter Sky Survey: A preliminary catalogue. Astron. Astrophys. 2018, 613, A58. [CrossRef]

197. Condon, J.J.; Cotton, W.D.; Greisen, E.W.; Yin, Q.F.; Perley, R.A.; Taylor, G.B.; Broderick, J.J. The NRAO VLA Sky Survey. Astrophys. J. 1998, 115, 1693-1716. [CrossRef]

198. Bock, D.C.J.; Large, M.I.; Sadler, E.M. SUMSS: A Wide-Field Radio Imaging Survey of the Southern Sky. I. Science Goals, Survey Design, and Instrumentation. Astrophys. J. 1999, 117, 1578-1593. [CrossRef]

199. Gaensler, B.M.; Landecker, T.L.; Taylor, A.R.; POSSUM Collaboration. Survey Science with ASKAP: Polarization Sky Survey of the Universe's Magnetism (POSSUM). In American Astronomical Society Meeting Abstracts \#215; American Astronomical Society: Washington, DC, USA, 2010; Volume 215, p. 470.13.

200. Norris, R.P.; Hopkins, A.M.; Afonso, J.; Brown, S.; Condon, J.J.; Dunne, L.; Feain, I.; Hollow, R.; Jarvis, M.; Johnston-Hollitt, M.; et al. EMU: Evolutionary Map of the Universe. Publ. Astron. Soc. Aust. 2011, 28, 215-248. [CrossRef] 
201. Jarvis, M.; Taylor, R.; Agudo, I.; Allison, J.R.; Deane, R.P.; Frank, B.; Gupta, N.; Heywood, I.; Maddox, N.; McAlpine, K.; et al. The MeerKAT International GHz Tiered Extragalactic Exploration (MIGHTEE) Survey. In MeerKAT Science: On the Pathway to the SKA; Sissa Medialab: Trieste, Italy, 2016; p. 6.

202. Blyth, S.; Baker, A.J.; Holwerda, B.; Bouchard, A.; Catinella, B.; Chemin, L.; Cunnama, D.; Davé, R.; Faltenbacher, A.; February, S.; et al. LADUMA: Looking at the Distant Universe with the MeerKAT Array. In MeerKAT Science: On the Pathway to the SKA; Sissa Medialab: Trieste, Italy, 2016; p. 4.

203. de Blok, W.J.G.; Adams, E.A.K.; Amram, P.; Athanassoula, E.; Bagetakos, I.; Balkowski, C.; Bershady, M.A.; Beswick, R.J.; Bigiel, F.; Blyth, S.L.; et al. An Overview of the MHONGOOSE Survey: Observing Nearby Galaxies with MeerKAT. In MeerKAT Science: On the Pathway to the SKA; Sissa Medialab: Trieste, Italy, 2016; p. 7.

204. Bailes, M.; Barr, E.; Bhat, N.D.R.; Brink, J.; Buchner, S.; Burgay, M.; Camilo, F.; Champion, D.J.; Hessels, J.; Janssen, G.H.; et al. MeerTime - the MeerKAT Key Science Program on Pulsar Timing. arXiv 2018, arXiv:1803.07424.

205. Johnston, S.; Karastergiou, A.; Keith, M.J.; Song, X.; Weltevrede, P.; Abbate, F.; Bailes, M.; Buchner, S.; Camilo, F.; Geyer, M.; et al. The Thousand-Pulsar-Array programme on MeerKAT - I. Science objectives and first results. Mon. Not. R. Astron. Soc. 2020, 493, 3608-3615. [CrossRef]

206. Wolleben, M.; Landecker, T.L.; Carretti, E.; Dickey, J.M.; Fletcher, A.; Gaensler, B.M.; Han, J.L.; Haverkorn,·M.; Leahy, J.P.; McClure-Griffiths, N.M.; et al. GMIMS: the Global Magneto-Ionic Medium Survey. In Cosmic Magnetic Fields: From Planets, to Stars and Galaxies; Strassmeier, K.G., Kosovichev, A.G., Beckman, J.E., Eds.; IAU Symposium, Cambridge University Press: Cambridge, UK, 2009; Volume 259, pp. 89-90. [CrossRef]

207. CHIME/FRB Collaboration; Amiri, M.; Bandura, K.; Berger, P.; Bhardwaj, M.; Boyce, M.M.; Boyle, P.J.; Brar, C.; Burhanpurkar, M.; Chawla, P.; et al. Astrophys. J. 2018, 863, 48. [CrossRef]

208. Shimwell, T.W.; Röttgering, H.J.A.; Best, P.N.; Williams, W.L.; Dijkema, T.J.; de Gasperin, F.; Hardcastle, M.J.; Heald, G.H.; Hoang, D.N.; Horneffer, A.; et al. The LOFAR Two-metre Sky Survey. I. Survey description and preliminary data release. Astron. Astrophys. 2017, 598, A104. [CrossRef]

209. Shimwell, T.W.; Tasse, C.; Hardcastle, M.J.; Mechev, A.P.; Williams, W.L.; Best, P.N.; Röttgering, H.J.A.; Callingham, J.R.; Dijkema, T.J.; de Gasperin, F.; et al. The LOFAR Two-metre Sky Survey. II. First data release. Astron. Astrophys. 2019, 622, A1. [CrossRef]

210. Stuardi, C.; O'Sullivan, S.P.; Bonafede, A.; Brüggen, M.; Dabhade, P.; Horellou, C.; Morganti, R.; Carretti, E.; Heald, G.; Iacobelli, M.; et al. The LOFAR view of intergalactic magnetic fields with giant radio galaxies. arXiv 2020, arXiv:2004.05169.

211. Smith, D.J.B.; Best, P.N.; Duncan, K.J.; Hatch, N.A.; Jarvis, M.J.; Röttgering, H.J.A.; Simpson, C.J.; Stott, J.P.; Cochrane, R.K.; Coppin, K.E.; et al. The WEAVE-LOFAR Survey. In Proceedings of the SF2A-2016: Annual Meeting of the French Society of Astronomy and Astrophysics, Lyon, France, 14-17 June 2016; Reylé, C., Richard, J., Cambrésy, L., Deleuil, M., Pécontal, E., Tresse, L., Vauglin, I., Eds.; French Society of Astronomy and Astrophysics: Paris, France, 2016; pp. 271-280,

212. Wayth, R.B.; Lenc, E.; Bell, M.E.; Callingham, J.R.; Dwarakanath, K.S.; Franzen, T.M.O.; For, B.Q.; Gaensler, B.; Hancock, P.; Hindson, L.; et al. GLEAM: The GaLactic and Extragalactic All-Sky MWA Survey. Publ. Astron. Soc. Aust. 2015, 32, e025. [CrossRef]

213. Hurley-Walker, N.; Callingham, J.R.; Hancock, P.J.; Franzen, T.M.O.; Hindson, L.; Kapińska, A.D.; Morgan, J.; Offringa, A.R.; Wayth, R.B.; Wu, C.; et al. GaLactic and Extragalactic All-sky Murchison Widefield Array (GLEAM) survey - I. A low-frequency extragalactic catalogue. Mon. Not. R. Astron. Soc. 2017, 464, 1146-1167. [CrossRef]

214. Hurley-Walker, N.; Hancock, P.J.; Franzen, T.M.O.; Callingham, J.R.; Offringa, A.R.; Hindson, L.; Wu, C.; Bell, M.E.; For, B.Q.; Gaensler, B.M.; et al. GaLactic and Extragalactic All-sky Murchison Widefield Array (GLEAM) survey II: Galactic plane $345^{\circ}<l<67^{\circ}, 180^{\circ}<l<240^{\circ}$. Publ. Astron. Soc. Aust. 2019, $36, \mathrm{e} 047$. [CrossRef]

215. Riseley, C.J.; Galvin, T.J.; Sobey, C.; Vernstrom, T.; White, S.V.; Zhang, X.; Gaensler, B.M.; Heald, G.; Anderson, C.S.; Franzen, T.M.O.; et al. The POlarised GLEAM Survey (POGS) II: Results from an All-Sky Rotation Measure Synthesis Survey at Long Wavelengths. arXiv 2020, arXiv:2005.09266.

216. Beardsley, A.P.; Johnston-Hollitt, M.; Trott, C.M.; Pober, J.C.; Morgan, J.; Oberoi, D.; Kaplan, D.L.; Lynch, C.R.; Anderson, G.E.; McCauley, P.I.; et al. Science with the Murchison Widefield Array: Phase I results and Phase II opportunities. Publ. Astron. Soc. Aust. 2019, 36, e050. [CrossRef] 
217. Ferrière, K.M. The interstellar environment of our galaxy. Rev. Mod. Phys. 2001, 73, 1031-1066. [CrossRef]

218. Heiles, C.; Haverkorn, M. Magnetic Fields in the Multiphase Interstellar Medium. Space Sci. Rev. 2012, 166, 293-305. [CrossRef]

219. Hales, C.A. Calibration Errors in Interferometric Radio Polarimetry. Astrophys. J. 2017, 154, 54. [CrossRef]

220. Eyles, R.A.J.; Birkinshaw, M.; Smolčić, V.; Horellou, C.; Huynh, M.; Butler, A.; Delhaize, J.; Vignali, C.; Pierre, M. The XXL Survey. XXXIX. Polarised radio sources in the XXL-South field. Astron. Astrophys. 2020, 633, A6. [CrossRef]

221. Asad, K.M.B.; Koopmans, L.V.E.; Jelić, V.; de Bruyn, A.G.; Pandey, V.N.; Gehlot, B.K. Polarization leakage in epoch of reionization windows - III. Wide-field effects of narrow-field arrays. Mon. Not. R. Astron. Soc. 2018, 476, 3051-3062. [CrossRef]

222. Smirnov, O.M. Revisiting the radio interferometer measurement equation. I. A full-sky Jones formalism. Astron. Astrophys. 2011, 527, A106. [CrossRef]

223. Bhatnagar, S.; Rau, U.; Golap, K. Wide-field wide-band Interferometric Imaging: The WB A-Projection and Hybrid Algorithms. Astrophys. J. 2013, 770, 91. [CrossRef]

224. Mevius, M.; van der Tol, S.; Pandey, V.N.; Vedantham, H.K.; Brentjens, M.A.; de Bruyn, A.G.; Abdalla, F.B.; Asad, K.M.B.; Bregman, J.D.; Brouw, W.N.; et al. Probing ionospheric structures using the LOFAR radio telescope. Radio Sci. 2016, 51, 927-941. [CrossRef]

225. Loi, S.T.; Trott, C.M.; Murphy, T.; Cairns, I.H.; Bell, M.; et al. Power spectrum analysis of ionospheric fluctuations with the Murchison Widefield Array. Radio Science 2015, 50, 574-597. [CrossRef]

226. Mannucci, A.J.; Wilson, B.D.; Yuan, D.N.; Ho, C.H.; Lindqwister, U.J.; Runge, T.F. A global mapping technique for GPS-derived ionospheric total electron content measurements. Radio Sci. 1998, 33, 565-582. [CrossRef]

227. Orús, R.; Hernández-Pajares, M.; Juan, J.M.; Sanz, J. Improvement of global ionospheric VTEC maps by using kriging interpolation technique. J.f Atmos. Sol. Terr. Phys. 2005, 67, 1598-1609. [CrossRef]

228. Malins, J.B.; White, S.M.; Taylor, G.B.; Stovall, K.; Dowell, J. Modeling the Ionosphere with GPS and Rotation Measure Observations. Radio Sci. 2018, 53, 724-738. [CrossRef]

229. Brentjens, M.A. Polarization Imaging with LOFAR; Astrophysics and Space Science Library: Springer: Basel, Switzerland, 2018; Volume 426, p. 159. [CrossRef]

230. Farnsworth, D.; Rudnick, L.; Brown, S. Integrated Polarization of Sources at $\lambda \sim 1 \mathrm{~m}$ and New Rotation Measure Ambiguities. Astrophys. J. 2011, 141, 191. [CrossRef]

231. O'Sullivan, S.P.; Brown, S.; Robishaw, T.; Schnitzeler, D.H.F.M.; McClure-Griffiths, N.M.; Feain, I.J.; Taylor, A.R.; Gaensler, B.M.; Land ecker, T.L.; Harvey-Smith, L.; et al. Complex Faraday depth structure of active galactic nuclei as revealed by broad-band radio polarimetry. Mon. Not. R. Astron. Soc. 2012, 421, 3300-3315. [CrossRef]

232. Hales, C.A.; Gaensler, B.M.; Norris, R.P.; Middelberg, E. Analytic detection thresholds for measurements of linearly polarized intensity using rotation measure synthesis. Mon. Not. R. Astron. Soc. 2012, 424, 2160-2172. [CrossRef]

233. Hales, C.A.; Murphy, T.; Curran, J.R.; Middelberg, E.; Gaensler, B.M.; Norris, R.P. BLOBCAT: software to catalogue flood-filled blobs in radio images of total intensity and linear polarization. Mon. Not. R. Astron. Soc. 2012, 425, 979-996. [CrossRef]

234. Farnes, J.S.; Heald, G.; Junklewitz, H.; Mulcahy, D.D.; Haverkorn, M.; Van Eck, C.L.; Riseley, C.J.; Brentjens, M.; Horellou, C.; Vacca, V.; et al. Source finding in linear polarization for LOFAR, and SKA predecessor surveys, using Faraday moments. Mon. Not. R. Astron. Soc. 2018, 474, 3280-3296. [CrossRef]

235. Bell, M.R.; Enßlin, T.A. Faraday synthesis. The synergy of aperture and rotation measure synthesis. Astron. Astrophys. 2012, 540, A80. [CrossRef]

236. Sridhar, S.S.; Heald, G.; van der Hulst, J.M. cuFFS: A GPU-accelerated code for Fast Faraday rotation measure Synthesis. Astron. Comput. 2018, 25, 205-212. [CrossRef]

237. Bell, M.R.; Oppermann, N.; Crai, A.; Enßlin, T.A. Improved CLEAN reconstructions for rotation measure synthesis with maximum likelihood estimation. Astron. Astrophys. 2013, 551, L7. [CrossRef]

238. Schnitzeler, D.H.F.M. Finding a complex polarized signal in wide-band radio data. Mon. Not. R. Astron. Soc. 2018, 474, 300-312. [CrossRef] 
239. Brown, S.; Bergerud, B.; Costa, A.; Gaensler, B.M.; Isbell, J.; LaRocca, D.; Norris, R.; Purcell, C.; Rudnick, L.; Sun, X. Classifying complex Faraday spectra with convolutional neural networks. Mon. Not. R. Astron. Soc. 2019, 483, 964-970. [CrossRef]

240. Pratley, L.; Johnston-Hollitt, M. Wide-band Rotation Measure Synthesis. arXiv 2019, arXiv:1906.00866.

241. Enßlin, T.A.; Frommert, M.; Kitaura, F.S. Information field theory for cosmological perturbation reconstruction and nonlinear signal analysis. Phys. Rev. D 2009, 80, 105005. [CrossRef]

242. Oppermann, N.; Junklewitz, H.; Robbers, G.; Bell, M.R.; Enßlin, T.A.; et al. An improved map of the Galactic Faraday sky. Astron. Astrophys. 2012, 542, A93. [CrossRef]

243. Oppermann, N.; Junklewitz, H.; Greiner, M.; Enßlin, T.A.; Akahori, T.; Carretti, E.; Gaensler, B.M.; Goobar, A.; Harvey-Smith, L.; Johnston-Hollitt, M.; et al. Estimating extragalactic Faraday rotation. Astron. Astrophys. 2015, 575, A118. [CrossRef]

244. Vacca, V.; Oppermann, N.; Enßlin, T.; Jasche, J.; Selig, M.; Greiner, M.; Junklewitz, H.; Reinecke, M.; Brüggen, M.; Carretti, E.; et al. Using rotation measure grids to detect cosmological magnetic fields: A Bayesian approach. Astron. Astrophys. 2016, 591, A13. [CrossRef]

245. Taylor, A.R.; Stil, J.M.; Sunstrum, C. A Rotation Measure Image of the Sky. Astrophys. J. 2009, 702, 1230-1236. [CrossRef]

246. Jelić, V.; Prelogović, D.; Haverkorn, M.; Remeijn, J.; Klindžić, D. Magnetically aligned straight depolarization canals and the rolling Hough transform. Astron. Astrophys. 2018, 615, L3. [CrossRef]

247. Clark, S.E.; Hensley, B.S. Mapping the Magnetic Interstellar Medium in Three Dimensions over the Full Sky with Neutral Hydrogen. Astrophys. J. 2019, 887, 136. [CrossRef]

248. Pratley, L.; Johnston-Hollitt, M. An improved method for polarimetric image restoration in interferometry. Mon. Not. R. Astron. Soc. 2016, 462, 3483-3501. [CrossRef]

249. Stil, J.M.; Keller, B.W.; George, S.J.; Taylor, A.R. Degree of Polarization and Source Counts of Faint Radio Sources from Stacking Polarized Intensity. Astrophys. J. 2014, 787, 99. [CrossRef]

250. Rudnick, L.; Owen, F.N. The Distribution of Polarized Radio Sources \&gt;15 $\mu$ Jy in GOODS-N. Astrophys. J. 2014, 785, 45. [CrossRef]

251. Braun, R.; Bonaldi, A.; Bourke, T.; Keane, E.; Wagg, J. Anticipated Performance of the Square Kilometre Array - Phase 1 (SKA1). arXiv 2019, arXiv:1912.12699.

252. Prandoni, I.; Seymour, N. Revealing the Physics and Evolution of Galaxies and Galaxy Clusters with SKA Continuum Surveys. In Advancing Astrophysics with the Square Kilometre Array (AASKA14); Sissa Medialab: Trieste, Italy, 2015; p. 67.

253. Rudnick, L. Optimizing Faraday Background Grids. arXiv 2019, arXiv:1901.09074.

254. Loi, F.; Murgia, M.; Govoni, F.; Vacca, V.; Prand oni, I.; Bonafede, A.; Feretti, L. Simulations of the polarized radio sky and predictions on the confusion limit in polarization for future radio surveys. Mon. Not. R. Astron. Soc. 2019, 485, 5285-5293. [CrossRef]

255. SKAO Science Team. SKA1 Level 0 Science Requirements. 2015. https://astronomers.skatelescope.org/wpcontent/uploads/2015/11/SKA-TEL-SKO-0000007_SKA1_Level_0_Science_RequirementsRev02-part-1signed.pdf (accessed on 4 June 2020).

256. Cantwell, T.M.; Bray, J.D.; Croston, J.H.; Scaife, A.M.M.; Mulcahy, D.D.; Best, P.N.; Bruggen, M.; Brunetti, G.; Callingham, J.R.; Clarke, A.O.; et al. Low-frequency observations of the Giant Radio Galaxy NGC 6251. arXiv 2020, arXiv:2004.11104.

257. Bonaldi, A.; Robert Braun for the SKAO Science Team. Square Kilometre Array Science Data Challenge 1. arXiv 2018, arXiv:1811.10454.

258. Sun, X.H.; Rudnick, L.; Akahori, T.; Anderson, C.S.; Bell, M.R.; Bray, J.D.; Farnes, J.S.; Ideguchi, S.; Kumazaki, K.; O’Brien, T.; et al. Comparison of Algorithms for Determination of Rotation Measure and Faraday Structure. I. 1100-1400 MHz. Astrophys. J. 2015, 149, 60. [CrossRef]

259. Van Eck, C.L.; Haverkorn, M.; Alves, M.I.R.; Beck, R.; de Bruyn, A.G.; Enßlin, T.; Farnes, J.S.; Ferrière, K.; Heald, G.; Horellou, C.; et al. Faraday tomography of the local interstellar medium with LOFAR: Galactic foregrounds towards IC 342. Astron. Astrophys. 2017, 597, A98. [CrossRef]

260. Van Eck, C.L.; Haverkorn, M.; Alves, M.I.R.; Beck, R.; Best, P.; Carretti, E.; Chyży, K.T.; Enßlin, T.; Farnes, J.S.; Ferrière, K.; et al. Diffuse polarized emission in the LOFAR Two-meter Sky Survey. Astron. Astrophys. 2019, 623, A71. [CrossRef] 
261. Bolton, R.C.; SRCCG. SKA Regional Centre Requirements. 2017. https:/ / astronomers.skatelescope.org/ wp-content/uploads/2017/10/SKA-TEL-SKO-0000735_01-SKA_Regional_Centre_Requirements.pdf (accessed on 4 June 2020).

262. An, T.; Wu, X.P.; Hong, X. SKA data take centre stage in China. Nat. Astron. 2019, 3, 1030. [CrossRef]

(c) 2020 by the authors. Licensee MDPI, Basel, Switzerland. This article is an open access article distributed under the terms and conditions of the Creative Commons Attribution (CC BY) license (http:// creativecommons.org/licenses/by/4.0/). 